\title{
Stiffnites. Part II
}

\author{
Maria Teresa Pareschi
}

Istituto Nazionale di Geofisica e Vulcanologia, Sezione di Pisa, Italy

\author{
Article history \\ Received October 2, 2009; accepted April 18, 2011. \\ Subject classification: \\ Submarine sediment flows, Magnetic fabric and anisotropy, Magnetic confinement, Shear polymer media, Triboelectric fragmentation, \\ Magnetic dipole Ambigua
}

\section{ABSTRACT}

The dynamics of a stiffnite are here inferred. A stiffnite is a sheet-shaped, gravity-driven submarine sediment flow, with a fabric made up of marine ooze. To infer stiffnite dynamics, order of magnitude estimations are used. Field deposits and experiments on materials taken from the literature are also used. Stiffnites can be tens or hundreds of kilometers wide, and a few centimeters / meters thick. They move on the sea slopes over hundreds of kilometers, reaching submarine velocities as high as $100 \mathrm{~m} / \mathrm{s}$. Hard grain friction favors grain fragmentation and formation of triboelectrically electrified particles and triboplasma (i.e., ions + electrons). Marine lipids favor isolation of electrical charges. At first, two basic assumptions are introduced, and checked a posteriori: (a) in a flowing stiffnite, magnetic dipole moments develop, with the magnetization proportional to the shear rate. I have named those dipoles as Ambigua. (b) Ambigua are 'vertically frozen' along stiffnite streamlines. From (a) and (b), it follows that: (i) Ambigua create a magnetic field (at peak, >1 T). (ii) Lorentz forces sort stiffnite particles into two superimposed sheets. The lower sheet, $\mathcal{L}^{+}$, has a sandy granulometry and a net positive electrical charge density. The upper sheet, $\mathcal{L}^{-}$, has a silty muddy granulometry and a net negative electrical charge density; the grains of sheet $\mathcal{L}^{-}$become finer upwards. (iii) Faraday forces push ferromagnetic grains towards the base of a stiffnite, so that a peak of magnetic susceptibility characterizes a stiffnite deposit. (iv) Stiffnites harden considerably during their motion, due to magnetic confinement. Stiffnite deposits and inferred stiffnite characteristics are compatible with a stable flow behavior against bending, pinch, or other macro instabilities. In the present report, a consistent hypothesis about the nature of Ambigua is provided.

\section{Introduction}

A stiffnite is a particular kind of gravity-driven submarine sediment flow that moves down-slope along the seafloor. In a stiffnite, the shear rate produces aligned magnetic dipoles, I have named these as Ambigua. In turn, Ambigua create a magnetic field, which sorts the electrified grains and groups ferromagnetic particles. The magnetic field of the Ambigua also confines and hardens a stiffnite body.
I believe that the hypothesis about magnetic dipole Ambigua and stiffnite dynamics is valid because it coherently and inexpansively explains the features of some kinds of marine deposits, which would otherwise require multiple, disjointed explanatory hypotheses.

The present report is organized as it follows: the Introduction, four central sections, and the Conclusions. Table 1 shows the list of the sections of this report, with their titles.

- In Section 2, I list the main characteristics of stiffnites and their dynamics.

- In Section 3, I describe some stiffnite deposits taken from the literature (although in the literature, these deposits are interpreted as turbidites).

- In Section 4, I describe a peak of magnetic susceptibility that occurs in stiffnite deposits taken from the literature (although, again, in the literature, these deposits are interpreted otherwise; e.g., as turbidite units). In this section, I also discuss the paramagnetic and ferromagnetic properties of the grains of a stiffnite deposit.

- In Section 5, I discuss and elaborate upon the stiffnite characteristics listed in Section 2. I do this in specific subsections. There are nine subsections in Section 5 in total. I check the hypotheses a posteriori. I use information/ constraints from field data on deposits, laboratory experiments on materials, and order-of-magnitude quantified estimations. I have taken the field data and experiments on materials from the literature.

\section{The stiffnite hypothesis}

A stiffnite is characterized by fine marine sediments. In the marine literature, the fine marine sediments of the sea floor are known as ooze. Hard fine particles of ooze are constituted, for example, by microshells, and in areas offshore of volcanic islands, by volcanic particles too. Polymers and other kinds of particles are also present.

Stiffnites are sheet shaped. They can be tens or hundreds of kilometers wide, and a few centimeters/few meters thick. In a reference coordinate system, let the positive $y$-axis be along 


\begin{tabular}{|c|c|}
\hline PARAGRAPH & SECTION (SUBSECTION) \\
\hline \multicolumn{2}{|l|}{ 1. Introduction } \\
\hline \multicolumn{2}{|l|}{ 2. The stiffnite hypothesis } \\
\hline \multirow[t]{4}{*}{ 3. In-field stiffnite deposits } & 3.1 Stiffnite deposits offshore of Hawaii \\
\hline & 3.2 Stiffnite deposits offshore of the Canary Islands \\
\hline & 3.3 Stiffnite deposits in the Horseshoe Abyssal Plain \\
\hline & 3.4 Stiffnites offshore of the mouth of the Tagus River \\
\hline \multirow[t]{4}{*}{ 4. The peak of magnetic susceptibility in stiffnite deposits } & 4.1 The size of the peak \\
\hline & 4.2 The magnetic properties of hard grains in stiffnite deposits \\
\hline & 4.2.1 Ferroan calcite of microshells are paramagnetic \\
\hline & 4.2.2 Ferromagnetic particles \\
\hline \multirow[t]{15}{*}{ 5. Constraints supporting stiffnite occurrence } & 5.1 In a stiffnite, grain fragmentation produces electrified particles and triboplasma \\
\hline & 5.2 The vertical profile of velocity in a mature stiffnite \\
\hline & 5.3 Magnetic dipole moments and magnetic field in a stiffnite \\
\hline & 5.3.1. First case study: One sheet-shaped conductor with current density $J$ \\
\hline & $\begin{array}{l}\text { 5.3.2. Second case study: Three sheet-shaped conductors with opposite but in-modulus equal } \\
\text { currents }\end{array}$ \\
\hline & 5.3.3. Third case study: Three sheets with different densities of magnetic dipoles \\
\hline & 5.3.4. A stiffnite with magnetization proportional to the shear rate \\
\hline & $\begin{array}{l}\text { 5.4 Magnetic Lorentz forces on electrified particles control the vertical grain size distribution } \\
\text { of a stiffnite }\end{array}$ \\
\hline & 5.5 The magnetic confinement of a stiffnite \\
\hline & 5.6 Triboplasma clouds and oxidation fronts \\
\hline & 5.7 The giant electric permittivity of a flowing stiffnite \\
\hline & 5.8 Magnetic Faraday forces explain the peak of magnetic susceptibility \\
\hline & 5.9 About the nature of stiffnite magnetic dipoles \\
\hline & 5.9.1 Preliminary considerations \\
\hline & 5.9.2 The nature of Ambigua \\
\hline
\end{tabular}

\section{Conclusions \\ Nomenclature \\ References}

Table 1.

the stiffnite down-slope motion, and the positive $z$-axis be along the reverse gravity, then the stiffnite tapering is along this $z$-axis.

A stiffnite is gravity driven along marine slopes and it undergoes shear (shear planes, $x$ - $y$ planes).

A stiffnite is formed by two sheets: (i) an upper, centimeter-to-meter-thick sheet, here named as $\mathcal{L}^{-}$, with a net negative electrical charge density; and (ii) a basal sheet that is a few centimeters to decimeters thick, here named as $\mathcal{L}^{+}$, with a net positive electrical charge density (Figure 1 ). The charges disappear in old stiffnite sediments at rest, due to ion diffusion and oxidation/reduction chemical reactions.

During motion, stiffnite grains are packed and exposed to shear. At tribolelectic contacts among the grains, stiffnite hard grains fragment and electrified particles and ions form. 'Tribo' is a Greek word that means 'to rub'. A triboelectric contact is a type of contact electrification in which certain materials become electrically charged after they come into contact with a different material and are then separated (e.g. after rubbing). The triboelectric scale lists materials

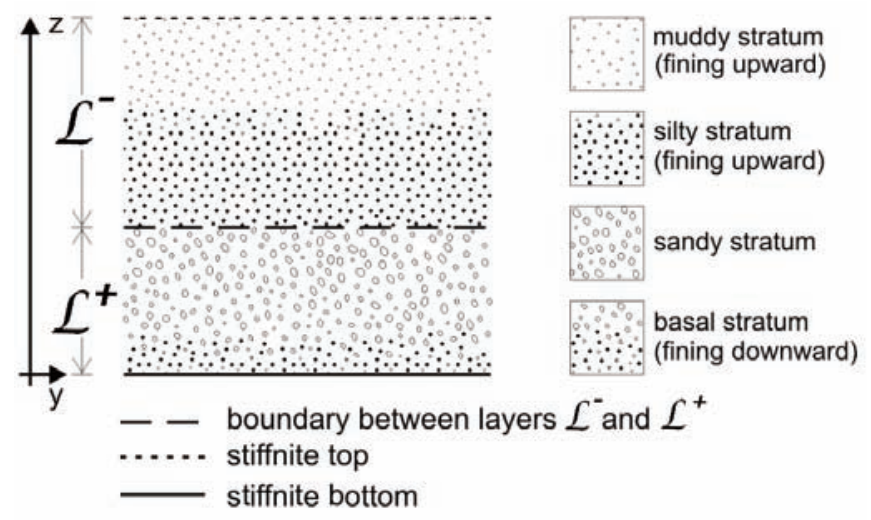

Figure 1. Illustration of a stiffnite deposit. The relative thicknesses of the various muddy, silty strata are not to scale. Sheet $\mathcal{L}$ is characterized by 'small' particles fining upwards. This overlies sheet $\mathcal{L}^{+}$, which is dominated by sandy particles. In the basal portion of sheet $\mathcal{L}^{+}$, there is a possible downwards fining of the grains. Large and small particles are identified in accordance to a radius $R_{t h}$, defined in the main text. In a flowing stiffnite, sheets $\mathcal{L}^{+}$and $\mathcal{L}$ have net positive and negative charge densities, respectively; they are neutral in an old stiffnite deposit at rest. Microshell fragments occur in the layers. 
according to their triboelectric properties [Shaw 1917]. Triboelectric effects also occur among dust particles of the same material, but of different sizes. When hard grains made of the same material slide or fragment, electrical charges are produced according to the triboelectric rules. In particular, at the sliding/breaking interface, smaller grains collect negative triboelectric electrons and bigger grains retain positive charges [Lacks and Levandovsky 2007]. In the present report, the same is inferred to occur to a fragmenting particle of a stiffnite: after fragmentation, the largest fragment retains the positive triboelectric charge, and the smaller fragments the negative polarities.

Calcite and aragonite are the main components of planktonic foraminifera and nannofossil micro-shells. The triboelectric properties of calcite and aragonite grains and of quartz and feldspar sands (including those from volcanic rocks) are well known [Shaw 1917]. Also, separation of triboelectricinduced charges is not unusual. Electromagnetic effects in Martian dust devils are related to triboelectric charge exchanges among small to large particles suspended in the atmospheric boundary layer, and these might affect future human occupation on Mars [Farrel et al. 2004]. In dust devils, water molecules reduce the charge separation [Farrel et al. 2004]. On the contrary, in stiffnites, the water effects appear to be reduced. Here, this is explained by the presence of marine lipids, which prevent tribolelectric charge from being 'washed away' by water molecules.

In a stiffnite body, magnetic dipoles $\vec{\mu}$ develop. I have named these dipoles as Ambigua. At first, some basic assumptions are introduced:

(i) Ambigua dipoles are aligned according to the positive $x$-axis. Ambigua create a magnetization $\vec{M}$ per unit volume, which is proportional to the shear rate. The shear rate increases from the top to the base of a stiffnite.

(ii) Ambigua are vertically frozen inside the flowing body of a stiffnite.

(iii) The deposit of a stiffnite gives a portrait of the particle distribution that occurs in a flowing stiffnite body.

These three hypotheses are checked a posteriori.

According to its definition, the magnetization $\vec{M}$ is the sum of all of the $N$ magnetic dipoles $\vec{\mu}$ per unit volume: $\vec{M}=$ $\vec{\mu} N$. The quantities $N, \vec{\mu}$ and $\vec{M}$ are functions of the vertical coordinate $z$, inside a stiffnite; these are null outside a stiffnite. The axes of magnetic dipoles $\vec{\mu}$ (i.e. of Ambigua) and of magnetization $\vec{M}$ are perpendicular to the stiffnite velocity, and lie in shear-rate planes.

The total magnetic moment $\vec{M}$ generates a magnetic field $\vec{B}_{\text {dip }}=\mu_{0} \vec{M}$, where $\mu_{0}$ is the permeability of the free space. For a flowing sheet of sediments, like a stiffnite moving down-slope along the $y$-axis, the magnetic field $B_{\text {dip }}$ is parallel to sea floor and points towards the left when looking at a stiffnite from its head (i.e. it is directed along the positive $x$-axis). $\vec{B}_{\text {dip }}$ has a maximum value at the base of a stiffnite; it is zero outside a stiffnite body.

Lorentz forces explain the grain sorting of a stiffnite deposit. The relation:

$$
\vec{F}_{L}=q \vec{u} \# \vec{B}
$$

tells us that a Lorentz force $\vec{F}_{L}$ acts on a particle, with an electrical charge $q$, moving at velocity $\vec{u}$ in an 'external' magnetic field $\vec{B}$.

The triboelectrically electrified grains of a stiffnite have a down-slope velocity $\vec{u}$ (the stiffnite velocity, along the $y$-axis; Figure 6i) and the 'external magnetic field' $\vec{B}$ is just the field $\vec{B}_{\text {dip }}$ provided by the Ambigua.

As the grains are largely packed and their main motion is down-slope (i.e. ca. horizontal, because of the gentle marine slopes), the Lorentz forces are vertical. However, these act in opposite directions, according to the sign of the electrical charge $q$ of each single grain. Under Lorentz forces, in a flowing stiffnite, when spaces or, 'voids', randomly open among the larger grains in the flowing stiffnite body, electrically positive grains creep downwards, and electrically negative ones upwards. As a whole, in a mature stiffnite, Lorentz forces induce a vertical, size-dependent stratification of the electrified grains. Two sheets form: sheet $\mathcal{L}^{-}$overlies sheet $\mathcal{L}^{+}$(Figure 1).

The grains of sheet $\mathcal{L}^{-}$have a muddy-silty grain size and they become finer from the bottom to the top (Figure 1). Sheet $\mathcal{L}^{+}$is mainly constituted by sandy grains. These $\mathcal{L}^{+}$and $\mathcal{L}^{-}$sheets have average net positive and negative charge densities, respectively.

Vertical sorting can be explained on an order-ofmagnitude scale by Lorentz forces that act like 'equivalent variations in grain mass density'. In negatively electrified grains, Lorentz forces act as these grains should have 'an equivalent decrease' in their mass density with decreasing dimensions and increasing (negative) charges. In positively electrified grains, Lorentz forces act as these grains should have 'an equivalent increase' in their mass density with decreasing dimensions and increasing (positive) charge.

The smallest and the most negatively electrified paramagnetic grains collect at the top of sheet $\mathcal{L}^{-}$; the smallest and the most positively electrified paramagnetic grains collect at the bottom of sheet $\mathcal{L}^{+}$.

In the present report, the peak in magnetic susceptibility of stiffnite deposits is explained through Faraday forces. A Faraday force acts on a magnetic grain (characterized by a ferromagnetic susceptibility) in a spatially variable magnetic field ( $B_{\text {dip }}$ in this case). Faraday forces push ferromagnetic grains downwards. However, the position of the peak of magnetic susceptibility does not necessarily coincide with the lower boundary of a stiffnite, because Lorentz and Faraday forces promote opposite directions for negative grains.

Inside a stiffnite, the magnetic dipole Ambigua create 'equivalent currents', which run up-slope, because of the 
upwards-decreasing shear rate. Like wires of currents, streams of equivalent currents attract each other inside a stiffnite, creating confining magnetic pressures.

Magnetic attraction (acting along the $z$-axis) contributes to confine and stabilize a stiffnite. Magnetic attraction preserves stiffnite tapering and hardens these flowing bodies. Hardening, magnetic fields and Ambigua disappear in stiffnites at rest, as there shear is null, and 'relaxation' occurs (almost everywhere).

Stiffnites can climb opposite slopes. For example, some hundreds of kilometers offshore of the Hawaiian Islands, the height $\Delta H$ of a climbed submarine relief was $500 \mathrm{~m}$ (site 842 in Garcia, 1996), which suggests stiffnite submarine velocities as high as $100 \mathrm{~m} / \mathrm{s}$ at the base of this climbed relief.

In the present report, a consistent hypothesis about the nature of Ambigua is provided too. This hypothesis explains:

- the nature of the Ambigua magnetic dipoles;

- the alignment of the Ambigua magnetic dipoles as perpendicular to the direction of motion and along the shear planes; and

- the 'vertically frozen' positions of Ambigua along stiffnite streamlines.

\section{In -field stiffnite deposits}

In this section, I describe some stiffnite deposits taken from the literature, although they were previously interpreted as turbidites. I have identified evidence of stiffnite deposits throughout the marine world: offshore of Hawaii and the Canary Islands, triggered by tsunamis due to past giant landslides that originated from flank collapses of these volcanic islands (Figures 2 and 3); offshore of the Tagus River, related to the 1755 Lisbon (Portugal) tsunami/earthquake (Figure 3a); in the Horseshoe and Tagus Abyssal Plains off the Portuguese coast (western Iberian margin; Figure 3a); and in the Madeira Abyssal Plains (North-East Atlantic; Figure3a). I discuss these in next sections (i.e. in Subsections 3.1, 3.2, 3.3 and 3.4).

I note that reports in the literature have interpreted the below-listed units as turbidite deposits (with a coarser base), derived, for example, «from a distal landslide front», or from «settling out from water currents», during flow sinking along continental margins or along submarine sea-mount slopes. These water currents «bring in suspensions particles, perhaps densely packed at the flow base». The interpretation that these are stiffnites is mine.

\subsection{Stiffnite deposits offshore of Hawaii}

Garcia [1996] described multiple, closely spaced, sandy horizons with sharp bases and gradational tops, with each stack separated from each other by normal pelagic sedimentation (Figure 2, core 842 ). The authors interpreted these as turbidite deposits that evolved from giant Hawaiian landslides, from different spatial sources, and of different ages.

For example, one of these 'turbidite' deposits (or the stiffnite deposits, in my interpretation) is $11 \mathrm{~cm}$ thick and

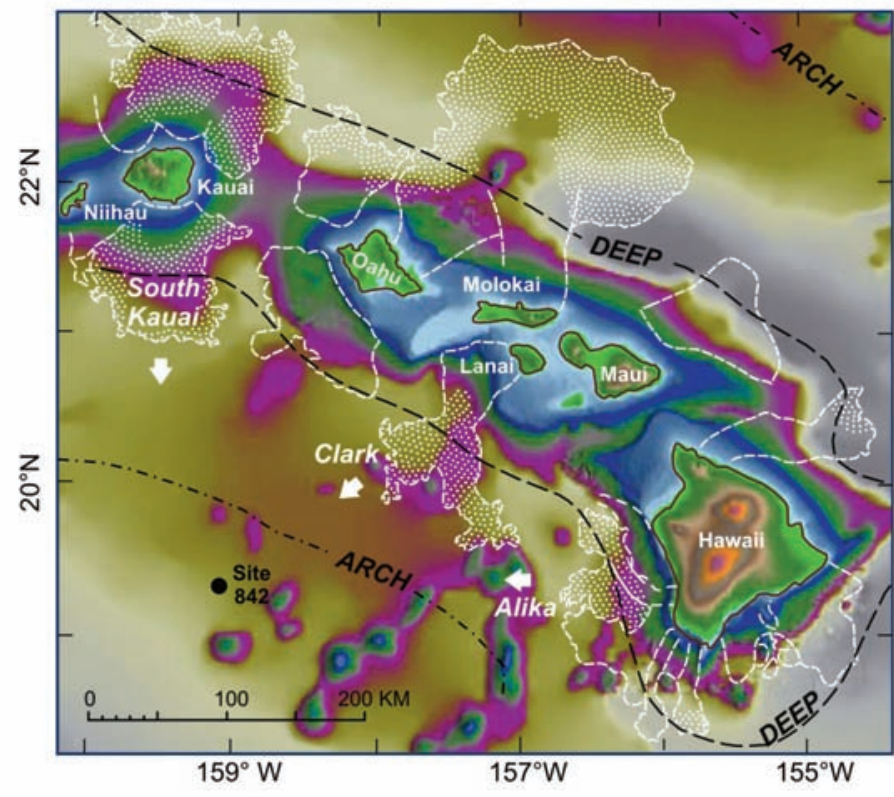

Figure 2. Map of Hawaiian Islands showing the major submarine landslides, indicated by white dashed lines and dots. Landslide deposits from Moore et al. [1989]. According to volcanic glass contents, site ODP 842 (solid black dot) shows traces of the Alika Slide from Mauna Loa Volcano (Hawaii Island), Clark Slide from Lanai, and the South Kauai Slide from Kauai [after Gracia 1999]. The axis of the Hawaiian Deep is shown by a heavy dashed line; the Hawaiian Arch axis is shown by the heavy dashed-dotted line. The difference of level between Site ODP 842 and the bottom of the Hawaiian Deep is about $500 \mathrm{~m}$. Colors of the bathymetric image are: medium blue lower limit marks $1000 \mathrm{~m}$ depth, dark blue lower limit marks $2000 \mathrm{~m}$, green lower limit marks $3000 \mathrm{~m}$, cyclamen lower marks limit $4000 \mathrm{~m}$, light olive-green lower limit marks $5000 \mathrm{~m}$, grey tones correspond to depths lower than $5000 \mathrm{~m}$. The bathymetric image is the file http://www.vterrain.org/Hawaii/Elevation/keating.jpg, downloaded from the web page of the Virtual Terrain Project (VTP): http: / / www.vterrain.org/Hawaii/Elevation/. It was produced by a group including: the Hawaii Mapping Research Group, the School of Ocean and Earth Science and Technology of the University of Hawaii at Manoa, the Hawaii Undersea Research Laboratory and the NOAA's Pacific Islands Benthic Habitat Mapping Center.

consists of many coarse/sand-rich-to-clayey subunits, which are less than $1 \mathrm{~cm}$ to a few centimeters in thickness [Garcia 1996]. In my interpretation, each subunit corresponds to a 'stiffnite pulse'. In all of these 'stacked' stiffnite deposits, sandy sheets alternate with muddy sheets, which suggests multiple pulses/multiple arrivals. The petrology of the grains indicates that they originated in the Hawaiian Islands or the close marine slopes (Figure 2) [Garcia 1996]. Moreover, the estimated ages of these deposits allow them to be roughly correlated with individual Hawaiian landslides [Garcia and Hull 1994, Garcia 1996, and references therein].

In my interpretation, at each tsunamigenic collapse of a volcanic island flank, soft marine soils were liquefied by a tsunami wave on the marine slopes, e.g., offshore of the collapsed volcanic island sector (Figure 2). As a consequence, at each collapse, stiffnites were triggered. From their wide sourcing areas, stiffnites moved on sea floor for hundreds of 
kilometers. Stiffnites reached the Hawaii Deep (Throat) and then they also climbed the relief of the Hawaii Arch, to reach site 842 (Figure 2). Different pulses (i.e. multiple stacked subunits for each collapse event at site 842) would suggest different stiffnite source areas, paths and travel times. The difference of the level climbed from the Hawaii Throat to site 842 is about $500 \mathrm{~m}$.

Garcia [1996] does not provide magnetic susceptibility data about these units.

\subsection{Stiffnite deposits offshore of the Canary Islands}

In my interpretation, a sequence of turbidites and stiffnites are interbedded with pelagic/hemipelagic sediments and can be recognized in giant piston cores in the North-East
Atlantic, offshore of Africa (Figure 3a, c), around the Canary Islands. Turbidite deposits are present too, which are characterized by a lack of peaks of magnetic susceptibility.

For these stiffnites, I believe that the same tsunami, related to a sector collapse of a volcanic Canary Island, liquefied fine marine sediments along: (i) the Canary Island submarine slopes; (ii) along the slopes of submerged marine releves (e.g., the Meteor Seamounts, West of the Madeira Abyssal Plain, and others); and (iii) along the African continental slopes (Figure 3).

Liquefaction/instability of fine marine sediments depend on tsunami wave heights and marine slope steepness in the flow-source region, among other factors [Pareschi et al. 2006]. Different stiffnite paths from the source slopes to

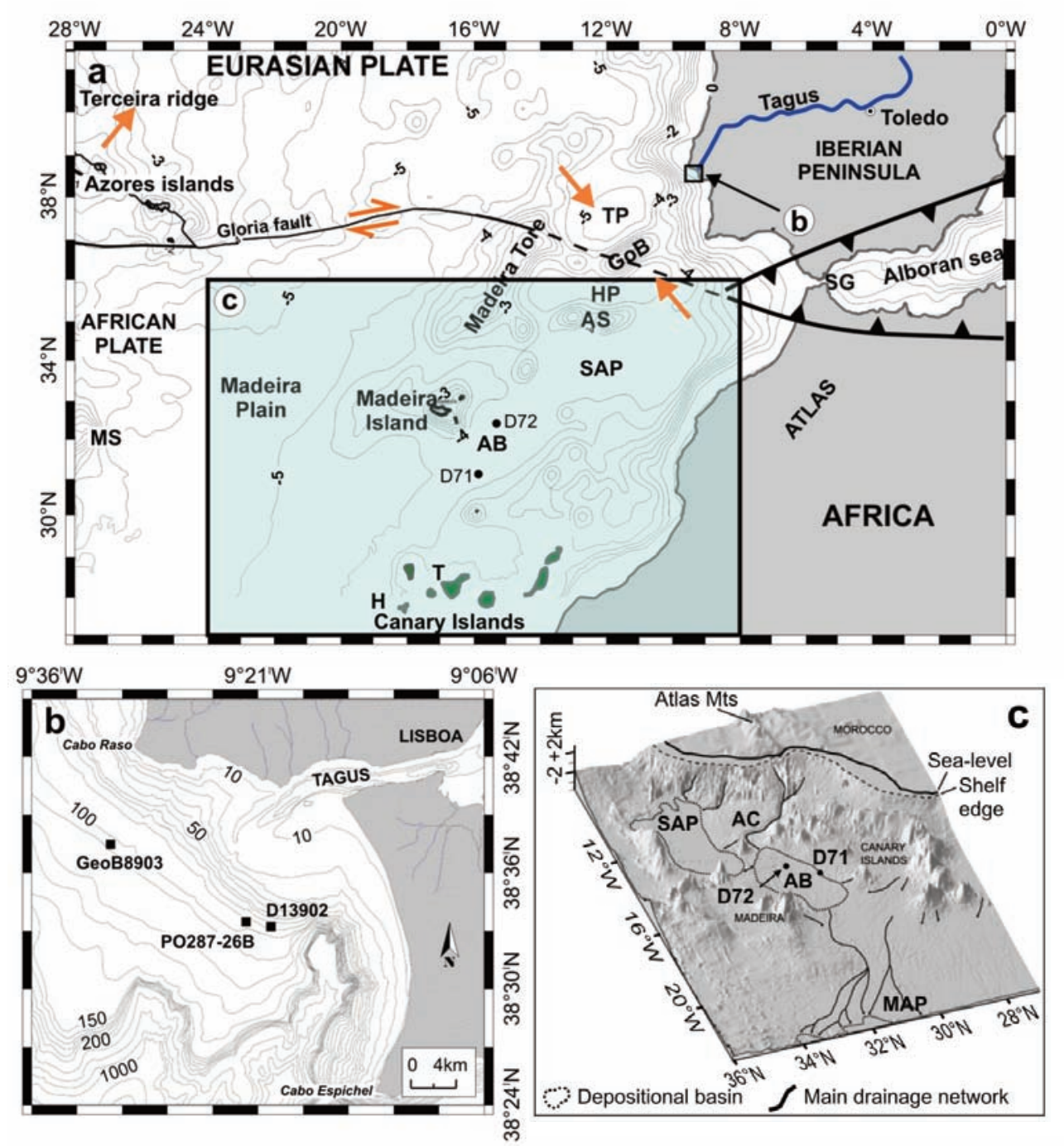

Figure 3. (a) Tectonic sketch and bathymetric map of the North-East Atlantic, with $500 \mathrm{~m}$ contour intervals. Orange arrows, plate convergence motion; orange semi-arrows, transform motion [after Morel and Meghraoui 1996, Jiménez-Munt et al. 2001]. Abbreviations: GB, Galicia Bank; TP, Tagus Plain; GoB, Gorrige Bank; HP, Horseshoe Plain; AS, Ampere Seamount; SAP, Seine Abyssal Plain; AB, Agadir Basin; SG, Strait of Gibraltar; H, El Hierro Island (Canaries); T, Tenerife Island (Canaries); MS, Meteor Seamounts. Rectangles b and c show the frames of panels (b) and (c) respectively. (b) Area offshore of the Tagur River estuary (West Iberian Margin); $10 \mathrm{~m}$ and $200 \mathrm{~m}$ contour intervals. Cores GeoB8903, D13902 and PO287-26B are mentioned in the main text [modified from Abrantes et al. 2005]. (c) Three-dimensional image of the NW African margin, showing the locations of the Canary Islands, the Agadir Basin (AG), the Madeira Abyssal Plain (MAP), and the Seine Abyssal Plain (SAP). Perspective view of panel (c) from Wynn and Masson (2003). Cores D71 and D72 of panels (a) and (c) are mentioned in the main text (modified from Wynn and Masson, 2003). 
the abyssal location originated many/some of the deposits described, for example, by Wynn et al. [2002], Wynn and Masson [2003] and Weaver et al. [1998].

Let us consider units $A B 2$ (also named as ' 2 ' or ' $b$ ') and $A B 14$ (also named as '14' or ' $g$ ') [Wynn et al. 2002]. These units can be correlated across a distance of more than 1000 $\mathrm{km}$, from the Seine Abyssal Plane to the Agadir/Seine levee, to the proximal and distal Agadir Basin and to the lower continental rise (Figure 3a) [Wynn et al. 2002]. For core location, see Wynn et al. [2002] (their Figures 6 and 8). Wynn et al. [2002] and Wynn and Masson [2003] interpret these as 'turbidite' deposits; I interpret these as stiffnite deposits.

Unit $b$ is dated offshore at about $15 \mathrm{Ka}$, and correlates with the El Golfo landslide [Wynn and Masson 2003], which originated from the failure of the north-east flank of El Hierro Island (Canary Islands; Figure 3a-c) [Masson 1996].

Unit $g$ is dated at about $170 \mathrm{ka}$, and correlates with the Icod landslide [Wynn and Masson 2003] on the north flank of Tenerife (Canary Islands; Figure 3a-c) [Watt and Masson 1995].

In cores D71 and D72 of the Agadir Basin, north of the Canary Islands (Figure 3a, c), Wynn and Masson [2003] reported that these deposits $b$ and $g$ show distinctive sequences of stacked subunits. I believe these are multiple superimposed stiffnites that are related to the same tsunamigenic island collapse. Wynn and Masson [2003] also reported that each subunit has a laminated sand/silt-rich base, becoming finer upwards, and separated by up to $10 \mathrm{~cm}$ of 'turbidite' (stiffnite, according to me) mud from the next subunit. The color and composition of each subunit are similar to those of the overlying and underlying ones, although towards the base of each of $b$ and $g$ deposit, the subunit generally has a coarser grain size. In my interpretation, each subunit corresponds to a different stiffnite pulse, although they are related to the same triggering tsunami. In particular, in my interpretation, the sand/silt-rich base of each subunit corresponds to an $\mathcal{L}^{+}$sheet, the muddy stratum to an $\mathcal{L}^{-}$sheet (Figure 1). In the stacked sequence, the muddy top of each $\mathcal{L}^{-}$sheet of a given stiffnite unit is in turn located below the $\mathcal{L}^{+}$sheet of another distinct, overlying, contiguous stiffnite unit.

As already said, for the same Canary Island collapse, I expect different stiffnites and corresponding different travel times, depending, for example, on the location where the tsunami waves liquefied the fine soft sediments: pelagic insular slopes, submarine pelagic releves, African shelves. I note that the potential of a tsunami wave (i.e. of a long wave) to liquefy sediments depends on the tsunami wave height, among other factorsr. In turn, the tsunami height is greater near the tsunami source, but the tsunami wave height increases with sea-floor shallowing too.

\subsection{Stiffnite deposits in the Horseshoe Abyssal Plain}

The Horseshoe Abyssal Plain is located on the western Iberian margin at an average depth of 4,800 $\mathrm{m}$ (Figure 3a). It is surrounded by topographic highs of about 3,000 m, with the exception of the Gorrige Bank, which rises to only $20 \mathrm{~m}$ below sea level (Figure 3a). In my interpretation, the abyssal plain is build up by normal pelagic sediment layers and turbidites and stiffnite units. Typical stiffnite deposits (previously confused with turbidite deposits) can be identified in many of the cores collected throughout the plain by Lebreiro et al. [1997]. They consist of a sandy silt base a few centimeters thick (sheet $\mathcal{L}^{+}$in my interpretation). Above this there is a muddy sheet (sheet $\mathcal{L}^{-}$in my interpretation), a decimeter to a meter thick. «The brownish color» of some of the thick stiffnites (turbidites, they say) «indicates oxidation in situ of the organic matter», which was originally characterized by 'olive-green' colors [Lebreiro et al. 1997].

For example, I interprete $\mathrm{H}_{2}, \mathrm{H}_{8}$, and $\mathrm{H}_{13}$ of Lebreiro et al. [1997] as stiffnite units. These are three thick beds of the order of one meter in thickness. Also the thinner beds of $\mathrm{H}_{9}$ and $\mathrm{H}_{10}$ are stiffnites. $\mathrm{H}_{9}$ and $\mathrm{H}_{10}$ are of the order of a few decimeters in thickness. For attribution, I use a peak of magnetic susceptibility that is easily visible in all of these deposits, as supported by cores collected in the Horseshoe Abyssal Plain, from East to West [Lebreiro et al. 1997; their Figure 4]. The data and nomenclature for $\mathrm{H}_{\mathrm{i}}$ are from Lebreiro et al. [1997].

All the thick beds of $\mathrm{H}_{2}, \mathrm{H}_{8}$, and $\mathrm{H}_{13}$ have colored oxidation fronts [Lebreiro et al. 1997]. On the contrary, the thick bed $\mathrm{H}_{3}$ is characterized by neither a magnetic susceptibility peak nor oxidations fronts; I interpret $\mathrm{H}_{3}$ as a true turbidite deposit.

\subsection{Stiffnites offshore of he mouth of the Tagus River}

Abrantes et al. [2008] reported some deposits that I interpret as stiffnite deposits. These are located in cores PO287-26B and D13902. These cores were collected by Abrantes et al. [2008] along the Portuguese continental shelf, offshore of the estuary of the Tagus River (Figure 3a, b). All of those deposits were triggered by the 1755 Lisbon earthquake [Abrantes et al. 2008].

The 1755 deposits of cores PO287-26B and D13902 are of the order of one decimeter in thickness, with a peaked grain size distribution. In both of these cores, the peak of grain size occurs at $400 \mu \mathrm{m}$, against background values of $50 \mu \mathrm{m}$; moreover, the peak in grain size is located some centimeters upwards from the peak of magnetic susceptibility [Abrantes et al. 2008]. Abrantes et al. [2008] sliced these cores at $1 \mathrm{~cm}$ intervals.

It is worth noting that the large values of hemipelagic sedimentation rates offshore of the mouth of the Tagus River $(>0.1 \mathrm{~cm} / \mathrm{yr}$, against pelagic sedimentation rates of some centimeters per kyr in the abyssal plains) allow the turbidites and stiffnite events that are inter-bedded by hemiplegics sedimentation strata to be well discriminated (and dated) over the last centuries. 


\section{The peak of magnetic susceptibility in stiffnite deposits}

Here I discuss the size of a peak of magnetic susceptibility in stiffnite deposits. This peak is an important feature of a stiffnite deposit, although it depends also on the availability of grains with ferromagnetic inclusions in the marine ooze. As a consequence, the peak of magnetic susceptibility can attain large values, e.g., in stiffnites starting from the marine slopes offshore of volcanic islands. Indeed, volcanic grains are usually rich in ferromagnetic components, like magnetite.

\subsection{The size of the peak}

In the literature, in stiffnite deposits (turbidite deposits, they say), quantitative values of magnetic susceptibility peaks are reported for example:

(i) offshore of the Canary Islands, for the stacked, abovementioned, deposits $b$ and $g$ [Wynn et al. 2002, Wynn and Masson 2003];

(ii) in the Horseshoe Abyssal Plain [Lebreiro et al. 1997], offshore of the Portuguese Margin, for deposits $\mathrm{H}_{2}, \mathrm{H}_{8}, \mathrm{H}_{9}$, $\mathrm{H}_{10}$ and $\mathrm{H}_{13}$, according to the nomenclature of Lebreiro et al. (1997);

(iii) and offshore of the Tagus river [Abrantes et al. 2008].

In my interpretation, these stiffnite deposits are possibly inter-bedded by strata of pelagic sedimentation and by 'true' turbidites.

In the following, I list the values of these peaks of magnetic susceptibility that occur in field stiffnite deposits. The background values refer to the magnetic susceptibility values far from the peak, which characterize pelagic sedimentation strata, for example.

In cores D13902, PO287-26B and GeoB8903, offshore of the mouth of the Tagus River (Portugal) (Figure 3a, b), Abrantes et al. (2008) reported magnetic susceptibility peaks of ca. $1500 \times 10^{-6} \mathrm{SI}$, ca. $1500 \times 10^{-6} \mathrm{SI}$ and ca. $500 \times 10^{-6} \mathrm{SI}$, respectively, for these stiffnites (turbidites, they say) that correlate with the 1755 Lisbon tsunami/earthquake [Abrantes et al. 2008]. These values are taken from Abrantes et al. [2008] (their Figures 2, 3 and 7) where, however, some typing errors occurred: i.e. the right scale factor should be $\times 10^{-6} \mathrm{SI}$ in their Figure 2, and $\times 10^{-5} \mathrm{SI}$ in their Figures 3 and 7 .

In these cores, the background values of magnetic susceptibility are listed in the following. In core GeoB8903 (Figure 3b), over the last $500 \mathrm{yr}$ the average background value is ca. $300 \times 10^{-6}$ SI. Before the Middle Ages, it was $<100 \times 10^{-6} \mathrm{SI}$ [Abrantes et al. 2008, their Figure 3]. In cores D13902 and PO287$26 \mathrm{~B}$ (Figure $3 \mathrm{~b}$ ), the average background values are ca. $500 \times$ $10^{-6}$ to $800 \times 10^{-6} \mathrm{SI}$ over the last $500 \mathrm{yr}$, and ca. $100 \times 10^{-6}$ to $200 \times 10^{-6} \times 10^{-6}$ SI before the Middle Ages [Abrantes et al. 2008].

Changes in the background values of the magnetic susceptibility across the Middle Age are possibly related to the sword industry of the city of Toledo, which is located on the Tagus River in central Spain (Figure 3a). This industry, which began to flourish in the Middle Ages, involved the pouring of large amounts of iron and steel powders into the Tagus River, surrounding the city.

Inote that the 1755 Lisbon tsunami occurred in the period characterized by greater background values of magnetic susceptibility in the marine sediments of the Tagus estuary.

In the (stiffnite) units $\mathrm{H}_{2}, \mathrm{H}_{8}, \mathrm{H}_{9}, \mathrm{H}_{10}$ and $\mathrm{H}_{13}$ of the Horseshoe Abyssal Plain (Figure 3a), Lebreiro et al. [1997] reported peaks of magnetic susceptibility of ca. 15 to $30 \times 10^{-6}$ cgs or more (the multiplicative factor of $10^{-6}$ was missed in their Fig. 4). Taking into account the conversion factor $4 \pi$ [Hummel 2000, p. 308], these peaks have sizes of ca. 190 to $380 \times 10^{-6} \mathrm{SI}$ in the SI system. The background values were ca. $130 \times 10^{-6}$ SI units.

Wynn et al. [2002] and Wynn and Masson [2003] reported magnetic susceptibility peaks of $500 \times 10^{-6} \mathrm{SI}$ in the $b$ and $g$ stacked stiffnite units in cores D71 and D72, collected in the Agadir Basin (Figure 3a, c). The background values were less than ca. $50 \times 10^{-6}$ SI. All of these values were reported in their Figure 6 and Figure 3 respectively, where, however, a typing error erased the multiplication factor $10^{-6}$ from the magnetic susceptibility scale of their figures.

\subsection{The magnetic properties of hard grains in stiffnite deposits}

Here, I briefly review the magnetic properties that characterize some kinds of the hard particles in stiffnite deposits.

Let me consider the magnetic properties of two kinds of grains in marine ooze, i.e. sandy-clay calcite microshells, and ferromagnetic grains. Here, 'magnetic particle' means that a magnetic moment develops in that particle in the presence of an external magnetic field. This magnetic moment is permanent in a 'ferromagnetic grain' (i.e. a grain with ferromagnetic inclusions).

\subsubsection{The ferroan calcite of microshells is paramagnetic}

Calcite is the most stable polymorph of calcium carbonate $\left(\mathrm{CaCO}_{3}\right)$. It is the primary constituent of the shells of marine organisms, e.g. «plankton (such as coccoliths and planktonic foraminifera), the hard parts of red algae, some sponges, brachiopoda, echinoderms, most bryozoa, and parts of the shells of some bivalves such as oysters and rudists» [from Wikipedia, 'Calcite']. Calcite is a common constituent of sedimentary rocks and of limestone in particular, much of which is formed from the shells of dead marine organisms. Calcite is also a constituent of volcanic rocks. For these reasons, calcite is a very common component of marine sediments.

Calcite crystals are birefringent. Another anisotropic characteristic of a calcite crystal is the tensor nature of its magnetic permittivity. In the case of a homogeneous medium, note that the relative magnetic permittivity (or permeability) $\mu_{r}$ is a scalar that is equal to $1+\chi_{m}$, where $\chi_{m}$ is the (scalar) magnetic susceptibility. The same nomenclature and 
relationships hold for an anisotropic medium, in which case, however, the magnetic parameters are second rank tensors, as mentioned above.

The mean magnetic susceptibility (scalar value) is the arithmetic average $\left(k_{1}+k_{2}+k_{3}\right) / 3$ of the eigenvalues of the magnetic susceptibility tensor (where $k_{1} \geq k_{2} \geq k_{3}$ ). The susceptibility difference, i.e. $\Delta k=k_{1}-k_{3}$ [Schmidt et al. 2006], gives a quantitative idea of anisotropy. $\Delta k$ is related to the shape factor $U_{s}$ by the formula $U_{s}=\left(2 k_{2}-k_{1}-k_{3}\right) / \Delta k$, so that a perfect oblate shape $\left(k_{2}=k_{1}\right)$ has $U_{s}=1$, and a perfect prolate shape $\left(k_{2}=k_{3}\right)$ has $U_{s}=-1$. The anisotropy of magnetic susceptibility of calcite is low (Schmidt et al., 2006; and references therein). The intrinsic diamagnetic susceptibility of pure calcite is $-12.09 \pm 0.5 \times 10^{-6} \mathrm{SI}$, and the susceptibility difference is $1.10 \pm 0.01 \times 10^{-6} \mathrm{SI}$ [Schmidt et al. 2006].

However, a small proportion of iron or ferromagnetic inclusions can displace calcium in the calcite lattice and induce/increase the paramagnetic susceptibility of a calcite crystal, affecting its magnetic properties. As suggested by Figure 4, the presence of iron impurities is not unusual in nannofossil calcite shells [Abineri 1999]. A crystalline calcium carbonate in which a very small portion of the calcium in the crystal lattice is displaced by iron is called ferroan calcite. Ferroan calcite has anisotropy properties and an average paramagnetic susceptibility of ca. $200 \times 10^{-6} \mathrm{SI}$ [Rochette 1988].

The macroscopic paramagnetic susceptibility of crystals/aggregates of calcite is isotropic, if these crystals/ aggregates are disorderly 'assembled' and randomly

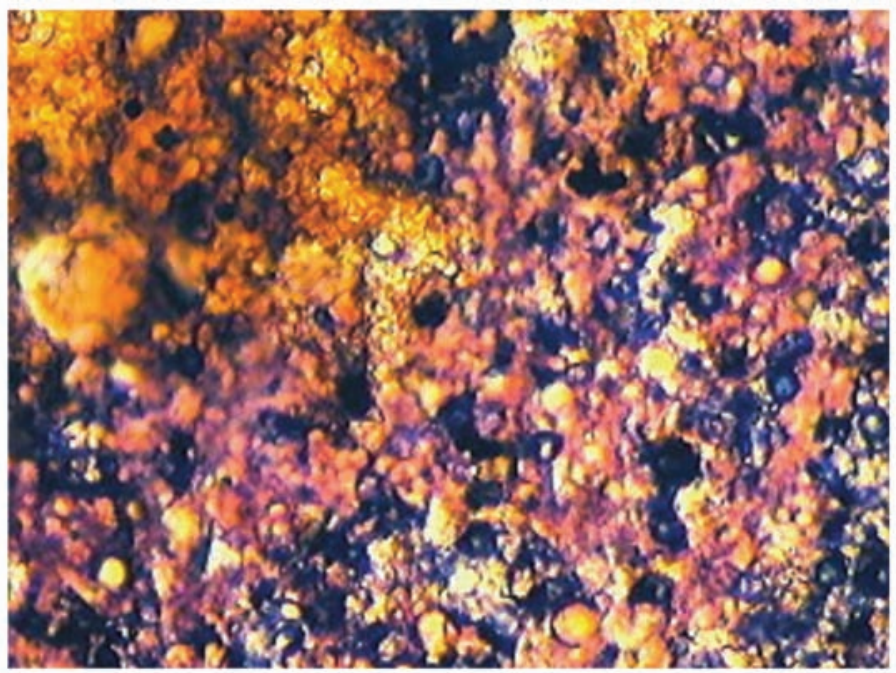

Figure 4. Differential laboratory staining of marine nannofossils (coccoliths and coccolithophores) in a layer of coccolithic limestone from the Upper Jurassic, Upper Kimmeridge Clay, Hounstout Cliff, Dorset coast (UK). The area of golden colored coccoliths is actually not stained, as these are buried in transparent kerogen. The pink nannofossils testify to a pure calcite composition. The dark blue nannofossils testify to a ferroan calcite composition, where $\mathrm{Fe}$ impurities are present in the calcite lattice (figure and comment from Abineri 1999). oriented in space.

As reviewed in the previous section, the average magnetic susceptibility $\chi_{m}^{\text {sh }}$ of pelagic sedimentation ooze strata constituted by ferroan-calcite/calcite microshells and other constituents, is ca. $100 \times 10^{-6}$ to $150 \times 10^{-6} \mathrm{SI}$ or even lower, with the exception of the Tagus River mouth (Figure $3 \mathrm{~b})$. Offshore of the Tagus River, hemipelagic sedimentation ooze strata have similar (low) average susceptibilities before the Middle Ages, and then larger values in the last 500 years. As already mentioned, this considerable increase in the background values since the Middle Ages is possibly due to ferromagnetic powders of anthropic origin that were discharged into the Tagus River by the iron/steel factories of Toledo.

A paramagnetic grain and assemblages of paramagnetic grains develop a temporary magnetic moment in the presence of an external magnetic field.

\subsubsection{Ferromagnetic particles}

Frequently, volcanic particles have an appreciable ferromagnetic fraction, such as inclusions of magnetite crystals. In this case, the single particle has moderate-to-high ferromagnetic properties.

Not-volcanic particles that are rich in ferromagnetic components can be found in stiffnite deposits (and marine sediments) too. The occurrence of steel powders in deposits is unusual, and this is related to local causes, as in the abovediscussed case of the Tagus River, Toledo.

The susceptibility $\chi^{f e}$ of a single ferromagnetic grain can be as high as $10^{-3} \mathrm{SI}$ or more, depending on the fraction of ferromagnetic components. As a reference, pure magnetite has a magnetic susceptibility of 1 to 10 SI [Lanza and Meloni 2006, p. 89]. For example, let the magnetite content of a grain be $1 \%$ in vol., and the other constituents of the grain have a magnetic susceptibility of $200 \times 10^{-6} \mathrm{SI}$. Then, the magnetite content has a determining role for the magnetic susceptibility of that grain, and the magnetic susceptibility of that grain is: $\chi^{\text {fe }}$ ca. $10^{-2}$ to $10^{-1} \mathrm{SI}$.

In the following, please read "ferromagnetic grain» as a shortening for "grain with an important fraction of ferromagnetic components so that $\chi^{f e}$ has typical values of $10^{-2}$ to $10^{-1}$ SI or higher».

As a consequence of its ferromagnetic components, a single grain acquires a permanent magnetic moment, if: (i) the grain is exposed to an external applied magnetic field; and (ii) the field is sufficiently high as to overcome the energy barrier related to the shape-dependent magnetostatic energy of magnetite or other ferromagnetic inclusions [Lanza and Meloni 2006, pp. 82-83].

An assemblage of grains with moderate-high ferromagnetic properties has a ferromagnetic behavior. Assemblages of ferromagnetic grains develop a (permanent) magnetic moment if an external magnetic field is applied. 


\section{Constraints supporting stiffnite occurrence}

In this section, the stiffnite features listed in Paragraph 2 are investigated. This section is organized into nine subsections. See Table 1 for the overall structures and titles of these subsections.

\subsection{In a stiffnite, grain fragmentation produces electrified particles and triboplasma}

In Pareschi [2011], it is shown that the microshells, and plausibly other thin or complex hard fine particles of different natures, can break during stiffnite motion. Here, I suggest that the sliding contact/fragmentation of hard grains produces electrified particles, i.e. charged grains and triboplasma, as positive and negative ions and electrons.

In the literature, this kind of contact that produces electrified particles is called triboelectric contact. With triboelectric contact, the polarity and strength of the charges produced differ according to the materials, surface roughness, temperature, strain and other properties. The associated charge exchange is a "quantum effect resulting from bringing the Fermi energy of two originally isolated grains to the same level upon contact) [Ashcroft and Mermin 1976]. According to the simple electronic theory of triboelectric phenomena, there are free electrons close to the surface of solids that can readily leave the surface when rubbed.

Simple rules regulate triboelectric exchanges: (i) a polished surface is always positively charged $(+)$ relative to a matt surface of the same material; (ii) a denser surface is always + relative to a less dense surface of the same material; (iii) dust from a body is always negatively charged (-) relative to the solid from which it derives [Shaw 1917]. Property (iii) was investigated recently in some detail by Lacks and Levandovsky [2007]. They showed that triboelectric charging in insulator systems composed of particles of identical material can be ascribed to the distribution of the particle sizes, such that smaller particles tend to charge negatively and larger particles tend to charge positively.

Recent experiments in nanotechnology [Nakayama and Martin 2006] have shown that a triboplasma cloud with an enormous number of electrons, ions and photons and electrified fragments is generated at the sliding contact between a 'hard' (a diamond) rounded tip and a hard plate, leeward of the running tip.

Triboelectric effects have been observed in many contexts: in field studies of volcanic plumes [Miura et al. 2002], sand storms and dust devils [Farrell et al. 2004]; and in laboratory experiments and industrial processes [Inculet et al. 2006].

By analogy, in the shear zone of a stiffnite, I infer that sliding among the grains and their fragmentation lead to the formation of small, electrically negative fragments and larger positive grains, and triboplasma (i.e. positive and negative ions and electrons).
Let me discuss the negligible effects of pore water molecules on these electrical charges. Farrell et al. [2004] reported that triboelectric effects in sand storms are enhanced by an arid climate, as the few water molecules, "cannot scavenge charges from the grains in these arid conditions».

In a marine environment, there are 'many' water molecules. However, in a stiffnite, the water molecules do not have any effects on the electrical charges.

To approach the enigma, let me describe the following experiment that was taken from the industrial literature; the methodology is proposed to separate calcite particles from quartz [Pearse and Pope 1976]. Oleate substances are known to help the triboelectric separation of quartz-calcite powders [Pearse and Pope 1976]. Experiments by Pearse and Pope [1976] showed that it is possible to efficiently separate quartzcalcite (and quartz-apatite) powders by triboelectric effects, after conditioning the mixture in aqueous oleate solutions and exposing it to an external electrical field. In more detail, in the method by Pearse and Pope [1976], calcite particles were separated from quartz by immersing them in an oleate floating solution, with sodium oleate and potassium hydroxide. The mixture was placed in a test-tube fitted with a side arm and bromoform was added slowly: the quartz floated off with the overflowing liquid, while the calcite remained submerged. Without chemical conditioning, these separations are impracticable. "Oleate adsorption has the advantage of making the mineral surface somewhat hydrophobic, so that triboelectric separations are virtually unaffected by changes in the ambient relative humidity» [from Pearse and Pope 1976].

What kind of oleate substances are there in a sea-floor environment? Many marine algae and other plankton species produce lipids. Each type of lipid has a slightly different structure, but they all have a large number of C-H bonds, which makes them a primarily nonpolar, energy-rich group of molecules. Another common characteristic of lipids is that they are generally insoluble in polar solvents, such as water. For example, the coccolithophore Emiliana huxleyi produces long-chain lipid molecules known as alkenones. Moreover, these lipid molecules can be detected in sediments, as alkenones are very resistant to decomposition and can survive millions of years [Volkman et al. 1998, and references therein].

By analogy with the experiments of Pearse and Pope (1976), I say that the oleate substances that allow triboelectric ion separations in stiffnites are marine lipids. I will return to this point further on in this report.

\subsection{The vertical profile of velocity in a mature stiffnite}

Here I propose vertical profiles for (horizontal) shear rates and flow velocities inside a stiffnite. A sheet-shaped sediment flow that moves down-slope is the reference geometry. The proposed velocity profiles will be checked a posteriori.

In a power-law generalized Newtonian fluid, there is a 
dependence of the shear stress $\tau$ on shear rate, according to the relation:

$$
\tau=\tau_{o}+K(\partial u / \partial z)^{\eta}
$$

where $K$ is the flow consistency index, $\tau_{\mathrm{o}}$ is the yield strength, $\eta$ is the flow behavior index, and $\partial u / \partial z$ is the shear rate. The shear stress $\tau$ is function of coordinate $z$ and it lies in an $x-y$ plane (the plane of shear); $z$ is the coordinate perpendicular to the sea floor, also known as the vertical coordinate because of the weak slope angles $\theta$ of marine inclines (usually, $\theta$ is a few degrees). The quantity:

$$
\mu_{e f f}=K(\partial u / \partial z)^{\eta-1}
$$

is the apparent or effective (if $\eta=1$ ) viscosity, as a function of the shear rate.

If, in Equation (2), $\eta$ is equal to 1.0, the flow is termed macro-viscous, or Bingham plastic. If $\eta$ is $<1.0$, the flow behavior is pseudoplastic or shear-thinning.

Pseudoplastic or shear-thinning flows $(\eta<1)$ have lower apparent viscosities at higher shear rates. They are usually solutions of large, polymeric molecules in a solvent with smaller molecules. The long polymeric molecules align themselves when they are sheared, and they slide along one another better once the shear increases. It is generally assumed that long molecular chains tumble at random and affect large volumes of fluid under low shear, but that they gradually align themselves to produce less resistance under high shear.

Some assumptions are introduced:

(i) the body is sheet shaped, of constant thickness $h$.

(ii) Stiffnite moves under the effect of gravitational forces: $\rho^{\prime} g(h-z) \sin \theta$, where $\rho^{\prime}$ is the submerged density of sediments (actual total density minus water density $\rho_{w}$ ).

(iii) Friction and drag can occur at stiffnite boundaries.

(iv) There is not a plug, i.e. all of the stiffnite body undergoes shear, or again, shear stress $\tau$ is always greater than the yield strength.

(v) The shear rate has a peak $s_{M}$ at $z=0$.
Under the above indicated assumptions and constitutive Equation (2), the flow moves down-slope, along the positive $y$ axis at a velocity $u(z)$, a function of the vertical coordinate $z$. Equations (4) and (5) provide the dependence of the horizontal (i.e. parallel to the sea floor) shear rate $d u / d z$ upon the vertical coordinate $z$, according to macrovisous $(\eta=1)$ and shearthinning $(\eta=1 / 2)$ behaviors, respectively (Figure 5a):

Macroviscous flow: $\frac{d u}{d z}=\frac{s_{M}}{h}(h-z) \quad$ in $0<z<h$

Shear-thinning $\quad \frac{d u}{d z}=\frac{s_{M}}{h^{2}}(h-z)^{2} \quad$ in $0<z<h$

Shear rate can also differ from zero at the top of the flow (Figure $5 \mathrm{~b}$ ).

The velocity profile of a macroviscous flow can be obtained by integrating Equation (4) on $z$ (Figure 5c). The same for the velocity profile of a shear-thinning flow.

Depth-averaged stiffnite velocities can reach high values. The Hawaiian stiffnite deposits at site 842 (Figure 2) [Garcia 1996] provide evidence of these high velocities. Stiffnites at site 842 are separated from each other by pelagic sedimentation, and each occurrence consists of a stack of some contiguous centimeter-thick stiffnite units. In other words, each subunit in a given stack. Characterized by its own peak of magnetic susceptibility, this is related to a different pulse of the same stiffnite, and following Garcia [1996], each stack is related to a well-defined tsunamigenic island collapse. Those collapses of Hawaii Islands were located hundreds of kilometers apart from each other and occurred millions of years from each other. They were: (i) the submarine tsunamigenic landslide Alika from Hawaii Island; (ii) Clark landslide from Lanai Island; and (iii) South Kauai landslide from Kauai Island [Garcia 1996, Moore et al. 1989] (Figure 2).

The correlated stiffnites sank down-slope of the Hawaiian slopes, crossed the Hawaiian Deep, and climbed the opposite slopes of the Hawaiian Arch, with a total submarine climb of $500 \mathrm{~m}$ (Figure 2). Their paths were hundreds of kilometers long.

At site 842 (Figure 2), the difference in height climbed
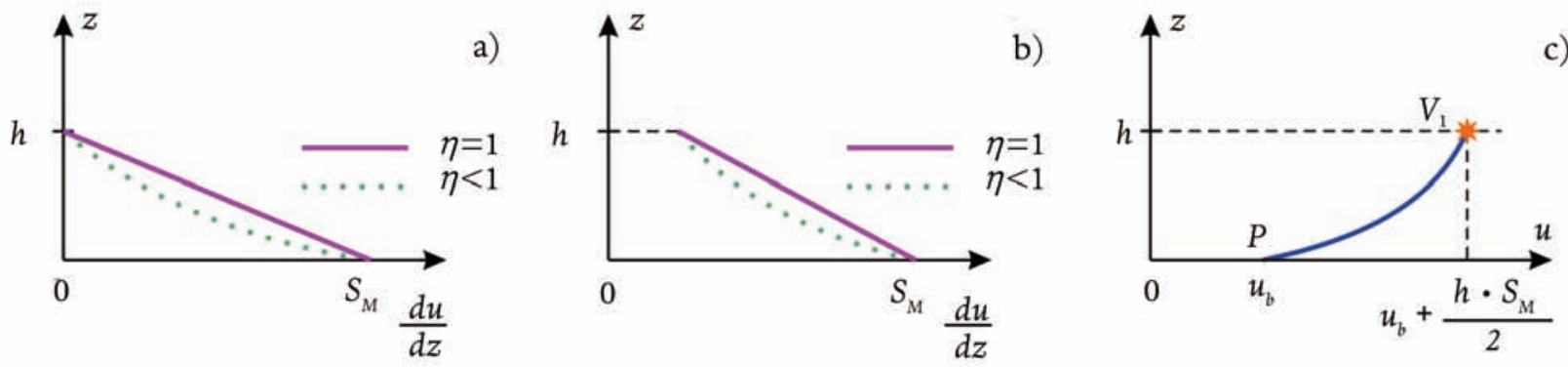

Figure 5. (a) Shear rate for a macro-viscous flow (cyclamen curve) and a shear-thinning flow (dotted green curve). Shear rate is null at the top of the flow and it has a peak $s_{M}$ at $z=0$. (b) The same as (a), but the shear rate differs from zero at the flow upper boundary. (c) Velocity profile of the macro-viscous flow of (a). The velocity profile is a parabola with vertex in $V_{1}$. At point $P$, the parabola has a tangent $=s_{M}$. 
a)

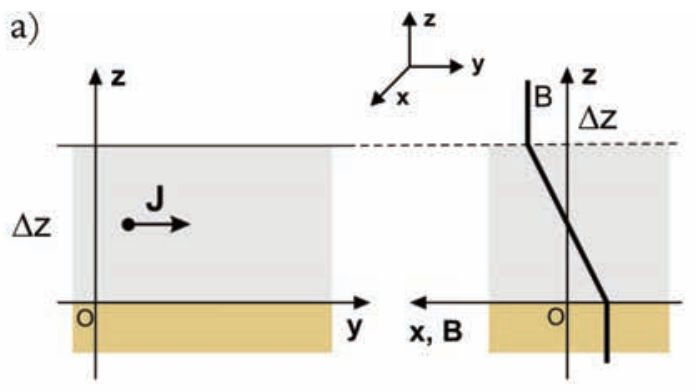

d)

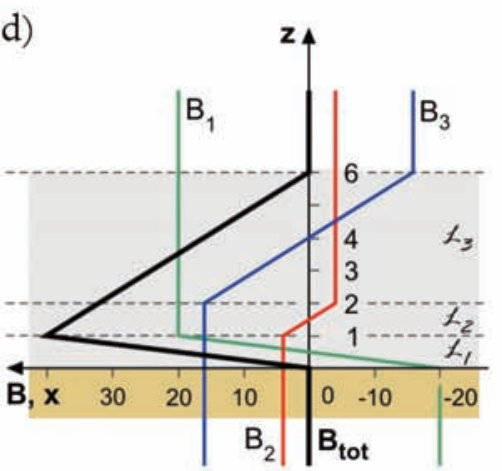

g)

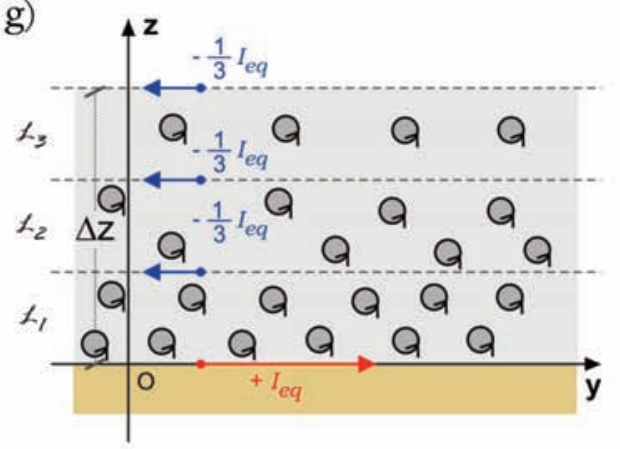

b)

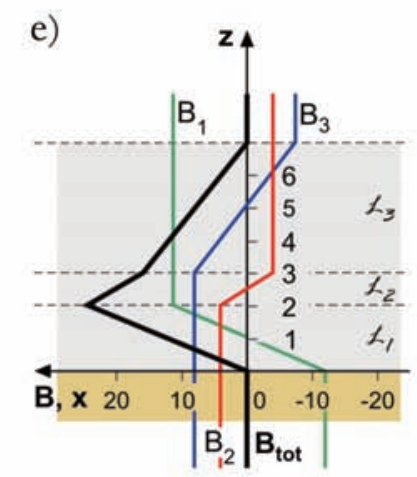

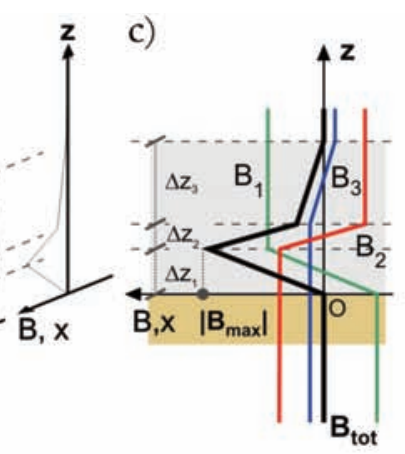

f)

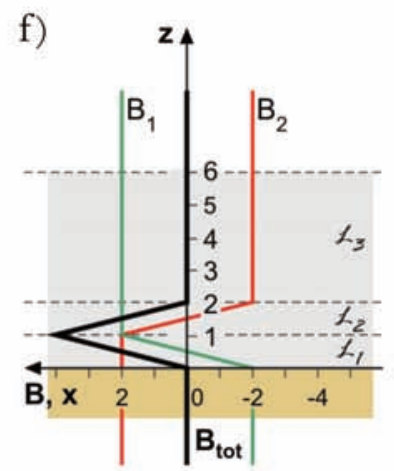

h)

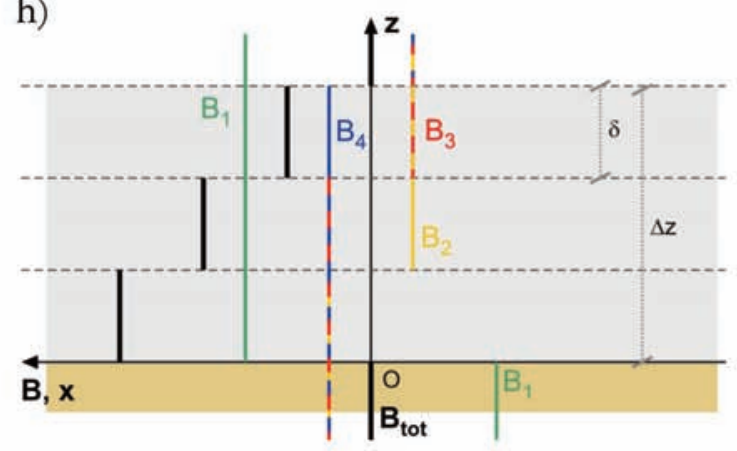

i)

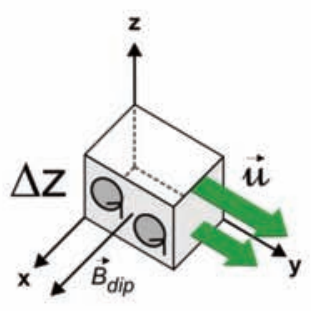

1)

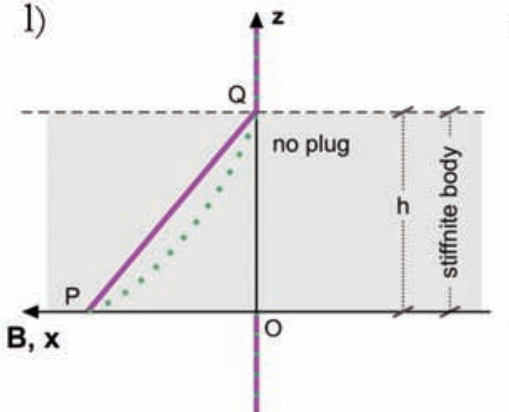

m)

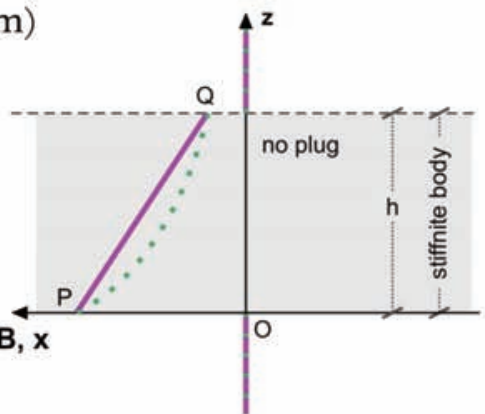

Shear thinning, convex profile, $\eta<1$;

Macro-viscous, linear profile, $\eta=1$

Figure 6. (a) In a conductor (gray) of thickness $\Delta z$, a current density $J$ (left) creates a magnetic field $B$ (right). (b, c) Conductor with three stacked sheets, from top to bottom: $\mathcal{L}_{1}, \mathcal{L}_{2}$ and $\mathcal{L}_{3}$, of thicknesses $\Delta z_{1}, \Delta z_{2}$ and $\Delta z_{3}$, respectively. In $\mathcal{L}_{1}$ there is a positive (along the positive $y$-axis) current density $J_{1}$, in $\mathcal{L}_{2}$ and $\mathcal{L}_{3}$ there are two negative current densities: $J_{2}$ and $J_{3}$, respectively. The three current densities create a positive magnetic field (black bold line) directed along the $x$-axis, with a peak at the interface between $\mathcal{L}_{1}$ and $\mathcal{L}_{2}$. The magnetic field is zero outside the conductors if Equation (7) holds. (d-f) The total magnetic field $B_{\text {tot }}$ (bold line) of cases 1, 2 and 3 of Table 2; green (red, blue) line is the magnetic field created by current density $J_{1}\left(J_{2}, J_{3}\right.$ respectively) running in $\mathcal{L}_{1}\left(\mathcal{L}_{2}, \mathcal{L}_{3}\right.$, respectively). Numerical values along the $x$-axis refer to magnetic field and they are multiples of reference units discussed in the main text and in the captions of Table 2. (g) Three stacked sheets with different densities of magnetic dipole moments. Dipole moments are parallel to the $x$-axis. They are generated by current loops (small circles with arrows showing current versus) lying in the $y$-z planes. Densities decrease bottom to top, but are constant inside each sheet. Red and green arrows are the equivalent currents created by these magnetic dipole moments. (h) Corresponding total magnetic field (bold line) in the plane $x$-z. Partial magnetic fields $B_{1}$ (green), $B_{2}$ (yellow), $B_{3}$ (red) and $B_{4}$ (blue), related to sheets $\mathcal{L}_{1}, \mathcal{L}_{2}$ and $\mathcal{L}_{3}$, are also shown. (i) Three-dimensional framework of a stiffnite, with the magnetic field $B_{\text {dip }}$, stiffnite velocity $u$ and magnetic dipoles (current loops) Ambigua. In the illustration, the Ambigua sizes are not to scale: in the real world, for example, Ambigua have a thread-like shape. $(1, \mathrm{~m})$ Illustrations of the magnetic field in a stiffnite flowing body (gray layer). Dotted and continuum lines show the magnetic field, according to different flow behaviors (see key-legend at the bottom). 
was $500 \mathrm{~m}$ at any flank collapse and stiffnite pulse. This suggests stiffnite submarine velocities as high as $100 \mathrm{~m} / \mathrm{s}$ in the Hawaiian Deep, at the base of the relief climbed. The formula velocity $\sim \sqrt{2 g \Delta H}$ has been used, where friction energy lost was neglected.

A 'normal' submarine mud flow with a yield strength of $1 \mathrm{kPa}$ to $100 \mathrm{kPa}$ of an electrostatic nature, cannot support flow velocities of tens of $\mathrm{m} / \mathrm{s}$. Indeed, the dynamic pressures of water on mud related to stiffnite velocities of $100 \mathrm{~m} / \mathrm{s}$, are ca. $1 / 2 \rho_{w} u^{2}=$ some MPa. This implies that stiffnites are 'particular' sediment flows, as we will also see in the next subsection.

\subsection{Magnetic dipole moments and magnetic field in a stiffnite}

Here I consider the magnetic field that arises inside a stiffnite, and its dipolar nature. In particular, in the first two subsections, I evaluate the effects of currents in sheet-shaped conductors. In the third section, I apply these formulae to the case of equivalent currents created by aligned magnetic dipoles. In the last subsection, I consider magnetic dipoles $\vec{\mu}$ (here called Ambigua), which are born inside a moving stiffnite, but disappear (almost everywhere) in a stiffnite at rest. Moreover, I evaluate the corresponding magnetic field by assuming that the total magnetization created by Ambigua is linearly proportional to the shear rate.

\subsubsection{First case study:}

One sheet-shaped conductor with current density J

A current with density $J$ running along the $y$-axis in a sheet-shaped conductor of thickness $\Delta z$, with its base at $z=0$, generates a magnetic field $B$ parallel to the $x$-axis, according to the following formulae (Figure 6a):

$$
\begin{gathered}
B=\frac{\mu_{0} \Delta z J}{2} \quad \text { for } z \geq \Delta z \\
B=-\mu_{0} J(0.5 \Delta z-z) \quad \text { for } 0 \leq z \leq \Delta z \\
B=-\frac{\mu_{0} \Delta z J}{2} \quad \text { for } z \leq 0
\end{gathered}
$$

where $\mu_{\mathrm{o}}$ is the permeability of free space $=4 \pi 10^{-7} \mathrm{Tm} / \mathrm{A}$.

\subsubsection{Second case study: Three sheet-shaped conductors} with opposite, but in-modulus, equal currents

Figure $6 \mathrm{~b}$ shows the case of three superimposed sheetshaped conductors. Let us say the three sheets are $\mathcal{L}_{1}, \mathcal{L}_{2}$ and $\mathcal{L}_{3}$ of thicknesses $\Delta z_{1}, \Delta z_{2}$ and $\Delta z_{3}$ and current densities $J_{1}, J_{2}$ and $J_{3}$ (Figure $6 \mathrm{~b}$ ). $J_{1}$ is positive, $J_{2}$ and $J_{3}$ are negative. Currents densities are along the $y$-axis. The suffix i refers to layer $\mathcal{L}_{i}$. I assume electrical current conservation:

$$
\left|\Delta z_{1} J_{1}\right|=\left|\Delta z_{2} J_{2}\right|+\left|\Delta z_{3} J_{3}\right|
$$

The total and partial magnetic fields created by current densities $J_{1}, J_{2}$ and $J_{3}$ are shown in Figure $6 \mathrm{c}$. The total magnetic field $B_{\text {tot }}$ is obtained by summing the contributions of the magnetic field created by each sheet, according to Equations (6) for a single sheet. Inside the set of sheets, the total magnetic field $\vec{B}_{\text {tot }}$ is always directed along the positive $x$-axis (Figure $6 \mathrm{~b}, \mathrm{c}$ ). At the interface between layers $\mathcal{L}_{1}$ and $\mathcal{L}_{2}, \vec{B}_{\text {tot }}$ has a peak $\left|\vec{B}_{\text {max }}\right|$ (towards the positive $x$-axis), of modulus:

$$
\left|\vec{B}_{\text {max }}\right|=\left|B_{1}\right|+\left|B_{2}\right|+\left|B_{3}\right|=\mu_{0} \Delta z_{1} J_{1}
$$

where:

$$
B_{1}=\frac{\mu_{0} \Delta z_{1} J_{1}}{2} ; B_{2}=\frac{\mu_{0} \Delta z_{2} J_{2}}{2} ; B_{3}=\frac{\mu_{0} \Delta z_{3} J_{3}}{2}
$$

Outside of the stacked layers (i.e. outside the conductor), the total magnetic field is zero, as a direct consequence of Equation (7).

Figures $6 \mathrm{~d}-\mathrm{f}$ and Table 2 show the case discussed above, by varying layer thicknesses and current densities. Layer thicknesses $\Delta z_{1}, \Delta z_{2}$ and $\Delta z_{3}$ are expressed as multiples of a given reference thickness $\delta$. Free charge densities $n_{1}, n_{2}$ and $n_{3}$ are expressed as multiples of a reference density $n$, and current densities $J_{1}, J_{2}$ and $J_{3}$ as multiples of a reference current density $J=V n q$. Charge $q$ is the elementary reference charge and $V$ is the reference velocity of charges, directed along the positive $y$ axis. Charges are positive in layer $\mathcal{L}_{1}$ and negative in layers $\mathcal{L}_{2}$

\begin{tabular}{c|c|c|c|c|c|c|c|c|c|c|c|c|c|c|c|c}
\hline & $V_{1}$ & $V_{2}$ & $V_{3}$ & $\Delta z_{1}$ & $\Delta z_{2}$ & $\Delta z_{3}$ & $n_{1}$ & $n_{2}$ & $n_{3}$ & $J_{1}$ & $J_{2}$ & $J_{3}$ & $\left|B_{1}\right|$ & $\left|B_{2}\right|$ & $\left|B_{3}\right|$ & $\left|B_{\max }\right|$ \\
\hline Units & $V$ & $V$ & $V$ & $\delta$ & $\delta$ & $\delta$ & $n$ & $n$ & $n$ & $n V q$ & $n V q$ & $n V q$ & $\mu_{\mathrm{o}} n q V \delta / 2$ & $\mu_{\mathrm{o}} n q V \delta / 2$ & $\mu_{\mathrm{o}} n q V \delta / 2$ & $\mu_{\mathrm{o}} n q V \delta / 2$ \\
\hline Case 1 & 1 & 1 & 1 & 1 & 1 & 4 & 20 & 4 & 4 & 20 & -4 & -4 & 20 & 4 & 16 & 40 \\
\hline Case 2 & 1 & 1 & 1 & 2 & 1 & 4 & 6 & 4 & 2 & 6 & -4 & -2 & 12 & 4 & 8 & 24 \\
\hline Case 3 & 1 & 1 & 1 & 1 & 1 & 4 & 2 & 2 & 0 & 2 & -2 & 0 & 2 & 2 & 0 & 4 \\
\hline
\end{tabular}

Table 2. Cases depicted in Figure 6d-f. In line 1, suffix 1 refers to the lowest sheet, suffix 2 to the intermediate sheet, suffix 3 to the top sheet. All of the quantities are expressed as multiples of reference values shown in the row of Units. $V, \delta, n, J$ and $B$ are respectively: charge velocity, sheet thickness, charge density, current density and magnetic field reference values (i.e. units). $V$ and $q$ are a reference velocity and unitary charge. $B_{\max }$ is the peak of the total magnetic field. $\mu_{\mathrm{o}}$ is the permeability of free space. 
and $\mathcal{L}_{3}$; consequently, the same is true for the current densities. Magnetic fields $B_{1}, B_{2}$ and $B_{3}$ are expressed as multiples of a reference field $B=\mu_{0} \delta V n q / 2$. See Table 2 for the estimations of the numerical values as multiples of the mentioned reference units.

The total magnetic field $B_{\text {tot }}$ shows a peak inside the conductors, at the interface between the positive and negative currents, and it is zero outside the conductors, on account of relation (7).

\subsubsection{Third case study: Three sheets with different densities} of magnetic dipoles

Let me now consider a body constituted by three sheets $\left(\mathcal{L}_{1}, \mathcal{L}_{2}\right.$ and $\left.\mathcal{L}_{3}\right)$, of comprehensive thickness $\Delta z$ and single-sheet thickness $\delta$, as that depicted in Figure $6 \mathrm{~g}$-h. Let us assume a constant magnetic dipole density in each sheet. Let the value of a single magnetic dipole moment be $\vec{\mu}$. Each magnetic dipole is created by a local current loop. Let the number of dipoles decrease from the bottom to the top layer. More precisely let $N$, $2 N / 3, N / 3$ be the densities of dipoles per unit volumes in each sheet, from the bottom to the top (Figure $6 \mathrm{~g}$, h). Figure $6 \mathrm{~g}$ depicts the small current loops associated with the dipole moments and the direction of the dipole currents. At the base of the body, the dipole currents create a total equivalent current $I_{e q}$ (per unit width), moving down-slope (positive $y$ axis). At the interior of each sheet, we see that each dipole current flowing in a given direction will be cancelled out by currents flowing in the opposite direction in the neighboring loops. The only place where cancellation does not take place is at the interfaces between the sheets, because of the different dipole densities (Figure $6 \mathrm{~g}$ ). The equivalent currents per unit width are (from bottom to top): $I_{e q}=N \mu$ at the base of the basal sheet $\mathcal{L}_{1} ;-I_{e q} / 3$ and $-I_{e q} / 3$ respectively at the interfaces of the two overlaying sheets (boundaries between $\mathcal{L}_{1}$ and $\mathcal{L}_{2}$ and between $\mathcal{L}_{2}$ and $\mathcal{L}_{3}$ ); again $-I_{e q} / 3$ at the top of the upper boundary of $\mathcal{L}_{3}$ (Figure $6 \mathrm{~g}$ ). According to the approach of Section 5.3.2, the total magnetic field (Figure 6h) can be computed by summing the contributions of each sheet. The total magnetic field is always positive inside the set of sheets and directed along the $x$-axis; moreover it shows a maximum value $B_{\max }=\mu_{0} I_{e q}=\mu_{0} \mu \mathrm{N}$, which is constant for the whole basal sheet $\mathcal{L}_{1}$. In the upper sheets, the magnetic field is constant inside each sheet, but the values decrease from a maximum in the basal sheet to zero in the medium above the sheets (Figure $6 \mathrm{~h})$. The magnetic field is null outside of the sheets.

\subsubsection{A stiffnite}

with magnetization proportional to the shear rate

Let me now assume that magnetic dipoles develop in the shear zone of a stiffnite, with magnetic dipole moments directed along the positive $x$-axis, i.e. towards the left looking at the down-flowing stiffnite from its head (Figure 6i). I have name those moments as Ambigua. These Ambigua develop in the entire body of a stiffnite. Indeed, according to the assumptions of Section 5.2, there in no plug in a mature stiffnite, and the entire stiffnite body undergoes the shear rate. In Figure 6i, the current loops are drawn as circles. However, they can have other shapes too; e.g., they can be connected tracks elongated along the $y$-axis. In this section, I evaluate the magnetic fields and the corresponding equivalent currents created by these magnetic dipoles. In another section, I will discuss the nature of hese dipoles.

The Ambigua magnetic dipoles create a total magnetization $\vec{M}(z)$ per unit volume, directed along the positive $x$-axis:

$$
\vec{M}(z)=N(z) \vec{\mu}(z)
$$

where $N(z)$ and $\vec{\mu}(z)$ are the density and the typical magnetic dipole moment of the Ambigua, respectively, at quote $z$. All of the quantities vary with $z \cdot \vec{M}(z)$ is directed along the positive $x$-axis, like the dipoles $\vec{\mu}$.

I assume that the magnetization $M(z)$ is proportional to the shear rate. Outside of a stiffnite, where the shear rate is zero and where there are no Ambigua, the magnetization is zero.

For simplicity, let me assume a stiffnite with a flow behavior index $\eta=1$ in Equation (2). According to Equation (4), the total magnetization is:

$$
M(z)=N_{M} \mu_{M} \frac{h-z}{h} \quad \text { in } 0<z<h
$$

where $N_{M}$ and $\mu_{M}$ are the values of $N(z)$ and $\mu(z)$, respectively, at $z=0$. Indeed, in Equation (11), I have assumed that the product of $N(z)$ times $\mu(z)$ have a linear trend and a peak at $z=0$.

A magnetization different from zero could also be possible at the top boundary of a stiffnite, according to the case of Figure $5 b$.

The total magnetization per unit volume creates a magnetic field :

$$
\vec{B}_{d i p}=\mu_{0} \vec{M}(z)=\mu_{0} N(z) \vec{\mu}(z)
$$

According to Equations (11) and (12), the magnetic field is always positive and is directed along the $x$-axis. Moreover, it has a peak $B_{\text {dip max }}$ at the stiffnite bottom boundary:

$$
B_{\text {dip } \max }=\mu_{0} \mu_{M} N_{M}
$$

Moreover, $\vec{B}_{d i p}$ is zero outside of the stiffnite body, where the magnetization is zero.

Figure 61 provides the profiles of the magnetic field according to the different velocity profiles (macroviscous and shear-thinning flows). Figure $6 \mathrm{~m}$ provides the profiles of the magnetic field, if the magnetization (and shear rate) differs 
from zero at the upper stiffnite boundary.

Now, let me derive Equation (13) further, and the trend of $\vec{B}_{\text {dip }}$ from the point of view of equivalent currents. Let us imagine a stiffnite as the superimposition of strata of infinitesimal thickness $d z$, characterized by a density of dipoles $N(z)$. On account of the decreasing magnetization, in the stiffnite body there is a constant current density per unit width along the $y$-axis (due to equivalent currents of magnetic dipoles):

$$
J_{e q}^{u p}(z)=\frac{\partial M}{\partial z}=-\frac{\mu_{M} N_{M}}{h}
$$

In Equation (14), $M$ is provided by Equation (11). The current density $J_{e q}^{u p}(z)$ creates a total equivalent current per unit width in the stiffnite body:

$$
I_{e q}^{u p}=\int J_{e q}^{u p}(z) d z=-N_{M} \mu_{M}
$$

The integral is performed from 0 to $h . I_{e q}^{u p}$ runs up-slope.

At the base of the stiffnite, according to the case study in Subsection 5.3.3 above, there is a positive equivalent current per unit width:

$$
I_{\text {eq }}^{\text {down }}=N_{M} \mu_{M}
$$

$I_{e q}^{\text {down }}$ runs down-slope. It is interesting to observe that the following relation holds:

$$
I_{e q}^{d o w n}=-I_{e q}^{u p}=N_{M} \mu_{M}
$$

Equation (17) guarantees that the magnetic field is zero outside the stiffnite. Moreover, extrapolating the case study in Subsection 5.3.3 above to the continuum, it follows that the total magnetic field increases linearly from the top to the bottom of a stiffnite; it is zero at the top, and it has a peak at the stiffnite bottom boundary, with a size of:

$$
B_{\text {dip } \max }=\mu_{0} I_{\text {eq }}^{\text {down }}=\mu_{0} \mu_{M} N_{M}
$$

This is the some result to that suggested by Equation (13). If the shear rate and the magnetization differ from zero at the top boundary of a stiffnite, it can be demonstrated that Equation (17) again holds. On account of Equation (17), the magnetic field is again zero outside a stiffnite.

Just to have a point of comparison, it is useful to provide the maximum possible strength of dipolar magnetization related to atoms, as evaluated by Haus and Melcher [1990]. Magnetic dipole moments of atoms typically have magnitudes of the order of $10^{-23} \mathrm{~A} \mathrm{~m}^{2}$. Typical atomic densities are $10^{29}$ atoms $/ \mathrm{m}^{3}$. Should all of the atomic dipole moments be aligned, then the magnetization due to atoms is $M$ ca. $10^{6} \mathrm{~A} / \mathrm{m}$. Because $\vec{B}=\mu_{0} \vec{M}$, the dipole moments of atoms create a maximum magnetic field of the order of $1 \mathrm{~T}$.

\subsection{Magnetic Lorentz forces on electrified particles control the vertical grain-size distribution of a stiffnite}

In this subsection, I demonstrate that the magnetic field of Figure 6l, or equivalently of Figure 6m, explains the grainsize distribution of a stiffnite deposit.

In nonelectrified granular flows, it is interesting to note that the larger grains move towards regions of lower shear rate, so that in many deposits of debris flows, an inverse grading is observed, with the smaller grains collected at the base of the deposit, and larger grains at the top [Savage and Lun 1988]. This is not the case of a stiffnite, even though a stiffnite is a sediment flow.

In Section 5.1, we saw that electrical charges are present inside a stiffnite, due to triboelectric effects.

According to Equation (1), the Lorentz force acting on an electrified grain is perpendicular to the velocity of that grain and to the magnetic field. As a consequence, inside a stiffnite, the Lorentz force displaces a given charged particle vertically if: (i) the particle is (quasi) packed inside the stiffnite body; (ii) the particle main motion occurs down-slope, with the particle quasi-integral with the stiffnite; (iii) the particle creeps 'slowly' along the vertical, inside the stiffnite itself; and (iv) this 'slowly' comes from the comparison with the 'fast' horizontal velocity of a stiffnite. In contrast to 'quasi'-packed grains, in an external magnetic field, a free particle moves along a circular path.

According to Equation (1), the magnetic force acting on an elementary charged particle is $1.6 \times 10^{-21} \mathrm{~N}, 1.6 \times 10^{-18} \mathrm{~N}$ and $1.6 \times 10^{-17} \mathrm{~N}$ for $B_{\text {dip }}=10^{-3} \mathrm{~T}, 1 \mathrm{~T}$ and $10 \mathrm{~T}$, respectively. Computations are carried out assuming: (i) one elementary charge $q=1.6 \times 10^{-19} \mathrm{C}$ per grain (the case of multiple charges can be easy extrapolated); and (ii) particles move down-slope within and integral with the stiffnite at a velocity of $10 \mathrm{~m} / \mathrm{s}$.

As the Earth magnetic field is ca. 2.5 to $6.0 \times 10^{-5} \mathrm{~T}$, magnetic forces related to $\vec{B}_{\text {dip }}$ can displace particles if the modulus of $\vec{B}_{\text {dip }}$ is much greater than $10^{-5} \mathrm{~T}$, from which my preliminary assumptions of $10^{-3} \mathrm{~T}, 1 \mathrm{~T}$ and $10 \mathrm{~T}$ arise.

Magnetic forces acting on micrometric electrically charged particles can vertically sort these particles according to their decreasing radius and polarity. Indeed, the action of the magnetic Lorentz force $F_{L}$ on a given charged grain can be considered equivalent to a variation in the gravity force $F_{g}$ on that grain, in the absence of the magnetic field $B_{\text {dip }}$. The density 'decreases' for an electrically negative charged particle, and 'increases' for a positively charged particle, with decreasing particle radius. In other words, these magnetic forces contrast or augment the gravity forces in negatively and positively electrified grains, respectively. As a whole, the sorting occurs because magnetic forces act as equivalent variations in the mass density of particles. Under the influence of magnetic forces and gravity, stiffnite grains reach the final stage of a stable stratified fluid.

The equivalent variation $\Delta \rho_{e q}$ of the density of a grain is: 


$$
\Delta \rho_{e q}=\rho_{g} F_{L} / F_{g}=\alpha q_{g} / r^{3}
$$

where $\rho_{g}$ is the mass density of the grain. Equation (19) shows that the equivalent variation $\Delta \rho_{e q}$ of a grain is proportional to its electrical charge $q_{g}$ and inversely proportional to the cube of the grain radius $r$. The smaller the radius of a particle, the greater (in absolute value) its equivalent variation in density. Table 3 helps us to quantitatively evaluate the effects of equivalent density variations for negative grains. The magnetic field $\vec{B}_{d i p}$ is $10^{-3}$ T, $1 \mathrm{~T}$ and $10 \mathrm{~T}$, respectively. Particles density is always $\rho_{g}=$ $1.2 \times 10^{3} \mathrm{~kg} / \mathrm{m}^{3}$; the particle radius varies according to Column 1 of Table 3 .

Columns 3, 4 and 5 of Table 3 provide the ratios between: (i) the magnetic Lorentz force $F_{L}$ acting on a particle; and (ii) the gravity force on that particle, in turn given by column 2 , for different values of $B_{\text {dip }}$. This ratio gives the relative equivalent variation in particle density. 'Equivalent' refers to the effect introduced by the magnetic Lorentz force $F_{L}$, which apparently changes the gravity force. 'Relative' refers to the dependence of $\Delta \rho_{e q}$ on the radius of the grain. For negative grains, Lorentz forces act as if the density of those grains decreased (reaching negative values) with decreasing grain size.

To estimate the vertical stability of the flowing body of a stiffnite with an 'equivalent' vertical density variation, I use the static stability, $E_{S S}$, for a vertical motionless stratified fluid, and the Richardson number, $R i$, for a stratified fluid with shear rate $d u / d z$ :

$$
E_{S S}=-\frac{1}{\rho} \frac{d \rho}{d z} \cong-\frac{\Delta \rho_{e q}}{\rho_{g}+\Delta \rho_{e q}} \frac{1}{\Delta z} \quad R i=g E_{S S} /(d u / d z)^{2}
$$

In the formula for $E_{S S}$, I have neglected the contribution of water to the equivalent density of a flowing stiffnite.

Columns 6, 7 and 8 of Table 3 give the static stability $E_{S S}$, for $B_{\text {dip }}=10^{-3} \mathrm{~T}, 1 \mathrm{~T}$ and $10 \mathrm{~T}$, respectively. To fix the computations, let us assume typical values of $\Delta z=10^{-1} \mathrm{~m}$. $\Delta z$ is a typical vertical length that is characterized by a variation in the grain sorting of a stiffnite and by changes in velocities. Columns 9, 10 and 11 of Table 3 give the Richardson number $R i$, for $B_{d i p}=10^{-3} \mathrm{~T}, 1 \mathrm{~T}$ and $10 \mathrm{~T}$ and shear rate $=10^{2} \mathrm{~s}^{-1}$.

Let us now evaluate stability conditions according to $R i$ and $E_{S S}$.

As mentioned, $R i$ estimates the dynamic stability that occurs in the presence of shear. In the inviscid limit (for water), the stable case occurs when $R i>0.25$. The higher viscosities of a stiffnite imply lower dynamic stability requirements. Indeed, a theoretical analysis by Defina et al.

\begin{tabular}{|c|c|c|c|c|c|c|c|c|c|c|}
\hline 1 & 2 & 3 & 4 & 5 & 6 & 7 & 8 & 9 & 10 & 11 \\
\hline \multicolumn{2}{|c|}{ Magnetic field (T) } & $10^{-3}$ & 1 & 10 & $10^{-3}$ & 1 & 10 & $10^{-3}$ & 1 & 10 \\
\hline \multicolumn{2}{|c|}{ Lorentz force $(\mathrm{N})$} & $1.610^{-21}$ & $1.610^{-18}$ & $1.610^{-17}$ & $1.610^{-21}$ & $1.610^{-18}$ & $1.610^{-17}$ & $1.610^{-21}$ & $1.610^{-18}$ & $1.610^{-17}$ \\
\hline \multirow{2}{*}{$\begin{array}{l}\text { Particle } \\
\text { radius } r\end{array}$} & \multirow{2}{*}{$\begin{array}{l}\text { Gravity } \\
\text { force }(\mathrm{N})\end{array}$} & \multirow{2}{*}{\multicolumn{3}{|c|}{$F_{L} / F_{g}=\Delta \rho_{e q} / \rho_{g}$}} & \multirow{2}{*}{\multicolumn{3}{|c|}{$\begin{array}{c}\text { static stability } E_{S S} \cong \\
-\frac{1}{\rho_{g}+\Delta \rho_{e q}} \frac{\Delta \rho_{e q}}{\Delta z}=-\frac{F_{L} / F_{g}}{1+F_{L} / F_{g}} \frac{1}{\Delta z}\end{array}$}} & \multirow{2}{*}{\multicolumn{3}{|c|}{$\begin{array}{l}R i=g E_{S S} /(d u / d z)^{2} \\
(\text { Richardson number })\end{array}$}} \\
\hline & & & & & & & & & & \\
\hline $1 \AA$ & $-5 \cdot 10^{-26}$ & $-10^{4}$ & $-10^{7}$ & $-10^{8}$ & -10 & -10 & -10 & $-10^{-2}$ & $-10^{-2}$ & $-10^{-2}$ \\
\hline $1 \mathrm{~nm}$ & $-5 \cdot 10^{-23}$ & $-10^{1}$ & $-10^{4}$ & $-10^{5}$ & -10 & -10 & -10 & $-10^{-2}$ & $-10^{-2}$ & $-10^{-2}$ \\
\hline $10 \mathrm{~nm}$ & $-5 \cdot 10^{-20}$ & $-10^{-2}$ & -10 & $-10^{2}$ & $10^{-1}$ & -10 & -10 & $10^{-4}$ & $-10^{-2}$ & $-10^{-2}$ \\
\hline $100 \mathrm{~nm}$ & $-5 \cdot 10^{-17}$ & $-10^{-5}$ & $-10^{-2}$ & $-10^{-1}$ & $10^{-4}$ & $10^{-1}$ & 1 & $10^{-7}$ & $10^{-4}$ & $10^{-3}$ \\
\hline $200 \mathrm{~nm}$ & $-4 \cdot 10^{-16}$ & $-10^{-6}$ & $-10^{-3}$ & $-10^{-2}$ & $10^{-5}$ & $10^{-2}$ & $10^{-1}$ & $10^{-8}$ & $10^{-5}$ & $10^{-4}$ \\
\hline $0.5 \mu \mathrm{m}$ & $-6 \cdot 10^{-15}$ & $-10^{-7}$ & $-10^{-4}$ & $-10^{-3}$ & $10^{-6}$ & $10^{-3}$ & $10^{-2}$ & $10^{-9}$ & $10^{-6}$ & $10^{-5}$ \\
\hline $1 \mu \mathrm{m}$ & $-5 \cdot 10^{-14}$ & $-10^{-8}$ & $-10^{-5}$ & $-10^{-4}$ & $10^{-7}$ & $10^{-4}$ & $10^{-3}$ & $10^{-10}$ & $10^{-7}$ & $10^{-6}$ \\
\hline $2 \mu \mathrm{m}$ & $-4 \cdot 10^{-13}$ & $-10^{-9}$ & $-10^{-6}$ & $-10^{-5}$ & $10^{-8}$ & $10^{-5}$ & $10^{-4}$ & $10^{-11}$ & $10^{-8}$ & $10^{-7}$ \\
\hline $5 \mu \mathrm{m}$ & $-6 \cdot 10^{-12}$ & $-10^{-10}$ & $-10^{-7}$ & $-10^{-6}$ & $10^{-9}$ & $10^{-6}$ & $10^{-5}$ & $10^{-12}$ & $10^{-9}$ & $10^{-8}$ \\
\hline $10 \mu \mathrm{m}$ & $-5 \cdot 10^{-11}$ & $-10^{-11}$ & $-10^{-8}$ & $-10^{-7}$ & $10^{-10}$ & $10^{-7}$ & $10^{-6}$ & $10^{-13}$ & $10^{-10}$ & $10^{-9}$ \\
\hline $20 \mu \mathrm{m}$ & $-4 \cdot 10^{-10}$ & $-10^{-12}$ & $-10^{-9}$ & $-10^{-8}$ & $10^{-11}$ & $10^{-8}$ & $10^{-7}$ & $10^{-14}$ & $10^{-11}$ & $10^{-10}$ \\
\hline $50 \mu \mathrm{m}$ & $-6 \cdot 10^{-9}$ & $-10^{-13}$ & $-10^{-10}$ & $-10^{-9}$ & $10^{-12}$ & $10^{-9}$ & $10^{-8}$ & $10^{-15}$ & $10^{-12}$ & $10^{-11}$ \\
\hline $100 \mu \mathrm{m}$ & $-5 \cdot 10^{-8}$ & $-10^{-14}$ & $-10^{-11}$ & $-10^{-10}$ & $10^{-13}$ & $10^{-10}$ & $10^{-9}$ & $10^{-16}$ & $10^{-13}$ & $10^{-12}$ \\
\hline $1 \mathrm{~mm}$ & $-5 \cdot 10^{-5}$ & $-10^{-17}$ & $-10^{-14}$ & $-10^{-13}$ & $10^{-16}$ & $10^{-13}$ & $10^{-12}$ & $10^{-19}$ & $10^{-16}$ & $10^{-15}$ \\
\hline
\end{tabular}
[1999] showed that a complete stabilization of the flow field

Table 3. Column numbers: column 1, grain radius; column 2, gravity force acting on a particle of a given radius and density $\rho_{g}=1.2 \times 10^{3} \mathrm{~kg} / \mathrm{m}^{3}$. Particles have a reference elementary charge $q=-1.6 \times 10^{-19} \mathrm{C}$. They move down-slope, integral with the stiffnite, at a velocity of $10 \mathrm{~m} / \mathrm{s}$. The magnetic field $B_{\text {dip }}$ and the correspondent Lorentz force $F_{L}$ acting on a grain are reported in rows 2 and 3 , for each column; columns $3,4,5$, ratios between the magnetic force $F_{L}$ and the gravity force of column 2 . Values of columns 3, 4, 5 times $\rho_{g}$ give the equivalent variation $=\Delta \rho_{e q}$ in the density of a grain; columns 6, 7, 8, static stability $E_{S S}$ for a stiffnite, evaluated by assuming a typical value of $\Delta z=10^{-1} \mathrm{~m}$; columns $9,10,11$, Richardson number $R i$, which measures dynamic stability in the presence of shear $\left(\right.$ here $\left.=100 \mathrm{~s}^{-1}\right)$. 
is attained with respect to infinitesimal disturbances, irrespective of the Richardson number, $R i$, as the Reynolds number, $R e$, decreases below 75 . In a stiffnite, Re is ca. smaller than 75 , if the density of the flow is $10^{3} \mathrm{~kg} / \mathrm{m}^{3}$, velocity $=10 \mathrm{~m} / \mathrm{s}(100 \mathrm{~m} / \mathrm{s})$, typical vertical length $=0.1 \mathrm{~m}$ and dynamic viscosity is greater than ca. $10 \mathrm{~Pa}$ s (100 Pa s). In these cases, and in the hypothesis of viscous behavior, the flow is stable because the low values of the Reynolds number $R e$, whatever the size of $R i$ in in columns 9-11 of Table 3.

Let me now discuss $E_{S S}$. Following the text by Stewart [2005, chapter 8.4], a fluid at rest is stable if $E_{S S}$ is greater than zero. The approximation used to derive $E_{S S}=-\rho^{-1} d \rho / d z$ is valid for $E_{S S}>50 \times 10^{-8} \mathrm{~m}^{-1}$. Moreover, in oceans, i.e., for static, stably stratified water, typical values of $E_{S S}$ are $5 \times 10^{-7} \mathrm{~m}^{-1}$ to $10^{-5} \mathrm{~m}^{-1}$ in the upper kilometer, and $E_{S S}$ is ca. $10^{-8} \mathrm{~m}^{-1}$ in deep trenches (depth $>7,000 \mathrm{~m}$ ). Let us consider the values of $E_{S S}$ in Table 3. For nanometric negatively charged particles, Lorentz forces dominate over gravity, whatever the value of $E_{S S}$ of Table 3, and those particles crowd towards the upper boundary of layer $\mathcal{L}^{-}$. For the other negative particles, from analogies with sea-water masses, stable conditions occur for $E_{S S}$ greater than $10^{-7} \mathrm{~m}^{-1}$ to $10^{-6} \mathrm{~m}^{-1}$. In Table 3, these values of $E_{S S}$ correspond to particle dimensions smaller than few tens of microns, if $B_{\text {dip }}$ ca. $1 \mathrm{~T}$ to $10 \mathrm{~T}$. We indicate this threshold in the particle radius by $R_{t h}$. As a whole, $R_{t h}$ distinguishes between large (coarse) and small grains. Magnetic forces act on small grains like an equivalent density variation, and a stable vertical sorting occurs for these small grains. For grains with a radius greater than $R_{t h}$, the magnetic forces are much too small with respect to those of gravity to produce internal vertical grain sorting of these large grains.

It is interesting to note that the threshold between fine sand and silt is some tens of microns, so that the $R_{t h}$ threshold can be materialized close to a well-defined granulometric classification limit.

Similar reasonings can be done for positively charged particles. For those positive particles, however, $\Delta \rho_{e q}$ is positive and $E_{S S}$ is always positive.

I imagine that in a stiffnite, vertical particle rearrangement is possible because fissures, or spaces, randomly open among larger grains during the downward motion of a stiffnite body. The smaller negatively charged particles creep upwards and the small positively charged grains creep downwards, through these 'voids'. Coarse (positive) grains $\left(>R_{t h}\right)$ «float» at an intermediate depth between positive and negative small grains.

In the field, stiffnite deposits appear to suggest that small positive grains are much less abundant than small negative grains, probably because at fragmentation, the smaller pieces retain a negative charge, and the larger pieces a positive charge, and so on, for new fragmentation of an already fragmented grain.

Electrical charges $q_{g}$ of grains can be larger than the elementary charge $q$. As a net result, in a mature stiffnite, the greater the negative electrical charge and the smaller the radius of a hard grain, the greater its distance from the stiffnite bottom boundary, i.e. the grain is located towards the top of $\mathcal{L}^{-}$. On the other hand, the greater the positive electrical charge and the smaller the radius of a hard grain, the closer it is to the stiffnite bottom boundary, i.e. the grain is located towards the bottom of $\mathcal{L}^{+}$(which coincides with the stiffnite bottom boundary).

I note the assumption that the vertical grain size distribution of a stiffnite deposit mimics that which occurs inside the corresponding flowing stiffnite body.

As seen in Section 3, stiffnite deposits from the literature (for example many deposits from Lebreiro et al. 1997) show a normal graded sheet of micrometric-sized grains (muddysilty sized grains) over a bed of coarser (sandy sized) grains. I recognize these strata respectively as sheet $\mathcal{L}^{+}$(nonsorted, sandy-sized grains) and sheet $\mathcal{L}^{-}$(upward fining, muddy-silty grains). When more alternating silty-muddy and sandy strata occur [Lebreiro et al. 1997], more stacked stiffnite units have to be evoked.

What about small $\left(<R_{t h}\right)$ positive grains in stiffnite deposits? In cores D13902 and PO287-26B (Figure 3b), Abrantes et al. [2008] (their Figure 2) described in detail a decimeters-thick stratum, with a central layer of sandy grains fining both downwards and upwards. This is the stiffnite correlated with the 1755 Lisbon tsunami/earthquake. In these patterns, I recognize a sheet $\mathcal{L}^{-}$, constituted by normal-graded small grains, and an underlying sheet $\mathcal{L}^{+}$, constituted by both an upper stratum of coarse grains and an underlying horizon with an inverse sorting.

\subsection{The magnetic confinement of a stiffnite}

In this section, I consider magnetic cohesion, i.e., the strength of the magnetic attraction that hardens the stiffnite body in the shear zone. Hardening occurs along the $z$-direction.

It is well known that wires carrying currents with the same directions attract each other; wires carrying currents of opposite directions repel each other. Like these wires, magnetic dipoles (or their equivalent currents) attract each other inside a stiffnite body. Indeed, magnetic dipoles create an equivalent current density running up-slope, according to Equation (14).

Let me broadly quantify the magnetic attraction (cohesion) along the vertical coordinate $z$. The magnetic force per unit area between two layers with current intensity $I_{1}$ and $I_{2}$ per unit width is:

$$
P_{m}=0.5 \mu_{0} I_{1} I_{2}
$$

Let me call $P_{m}$ the 'magnetic cohesion'. $P_{m}$ acts perpendicularly to the layers, i.e., along the $z$-axis, in the case of stiffnite sheets. Moreover, note that magnetic attraction 
does not depend on the distance between the layers.

To give some estimation, let us consider, for example, a reference sub-layer sited at the base of a stiffnite. Let this sublayer have a thickness $\delta$ equal to (say) one fifth of the thickness $h$ of the stiffnite (i.e., $\delta / h=0.2$ ). According to Equations (14), (18) and (21), it follows that this layer is under a confining pressure $P_{m}=0.5\left(B_{\text {dip } \max }\right)^{2} \delta(h-\delta) /\left(h^{2} \mu_{0}\right) \sim$ $0.5\left(B_{\text {dip } \max }\right)^{2} \delta /\left(h \mu_{0}\right) \sim 0.1$ to $10 \mathrm{MPa}$, if $B_{\text {dip } \max }=1 \div 10 \mathrm{~T}$.

Shear strengths of $1 \mathrm{MPa}$ characterize concrete, shear strengths of ca. $10 \mathrm{MPa}$ characterize wood, and $100 \mathrm{MPa}$ is the shear strength of marble. Stiffnite strengths of $1 \mathrm{MPa}$ to $10 \mathrm{MPa}$ mean that a stiffnite has a cohesion (along $z$ ) like that of wood.

Let us discuss the stabilizing effects of magnetic cohesion a bit. It is well known that the Kelvin-Helmholtz instability acts at the boundary between two fluids with different velocities. At this boundary, perturbations can grow, and this causes the disruption of a flowing sheet.

In particular, in the inviscid case and without surface tension, the instability amplifies exponentially, without control as the wave numbers $k \rightarrow \infty$ (Hadamard instability). If viscosity is present, small waves still grow, but their growth is limited and their amplitude does not go to infinity as $k \rightarrow \infty$ [Funada and Joseph 2001]. If the viscosity of one of the two fluids is much greater than the other one, then the growth factor is [after Funada and Joseph 2001]: ca. $1 / 2 \rho_{w} \Delta U^{2} / \mu_{S T}$, where $\Delta U$ is the difference of velocities. Let us say: $\Delta U=$ stiffnite velocity $u$ (because the sea water or the sediments of the sea floor are at rest), and $\mu_{S T}$ is the stiffnite viscosity at the stiffnite boundaries. The thin nature of stiffnite deposits, together with the observations that stiffnites can run along the sea floor for hundreds of kilometers, indicate that no boundary instabilities occur. It follows that in this flow approximation, the growth rate factor of small wave lengths must be zero. Consequently $\mu_{S T}$ has to be very, very large $(\rightarrow \infty)$. For example, according to the formula of Funada and Joseph [2001], the growth rate factor is less than/equal to 1.0 (but still acts), if $\Delta U=100 \mathrm{~m} / \mathrm{s}$ and $\mu_{S T}=10^{7} \mathrm{~Pa}$ s at the stiffnite upper boundary. Note that the viscosity of pitch is $\sim 10^{8} \mathrm{~Pa}$ s. According to Equation (3), in a shear-thinning flow $(\eta<1)$, the viscosity goes to $\infty$ as the shear rate goes to zero. A null shear rate at stiffnite boundaries (Figures 5a and 6l) is then compatible with a thinning stable flow behavior.

Alternatively, or in addition, if the shear rate is not null at the stiffnite boundaries (Figure $5 \mathrm{~b}$ ), magnetization occurs there with a related magnetic field (Figure $6 \mathrm{~m}$ ) and a confining magnetic pressure. I would imagine that a confining magnetic pressure acts as a sort of surface tension, and indeed, according to Funada and Joseph [2001], surface tension dumps instabilities.

\subsection{Triboplasma clouds and oxidation fronts}

Sliding contacts among the hard grains produce charged fragments and triboelectric clouds of ions and electrons [Nakayama and Martin 2006, Nakayama 2007]. By analogy, I imagine that the sliding of the hard marine grains inside a stiffnite produces charged fragments and triboplasma.

The typical dimensions of triboplasma particles are $\leq 1$ $\mathrm{nm}$, much smaller than average fragment dimensions. On average, some ions and electrons of triboplasma are expected to move vertically, under the effects of the magnetic field $\vec{B}_{\text {dip }}$, through the spaces in the assemblage of packed grains. Positive ions creep downwards, negative ions and electrons, upwards, whatever their initial positions inside the stiffnite. Some/many of these ions are expected to 'die' on account of chemical reactions at the surface of the stiffnite grains, when the triboplasma crashes against them.

Some stiffnite deposits show internal (i.e. inside the stiffnite body) «relicts of oxidation fronts, associated with metal concentrations and redox-related mobilization of trace elements». For example, this is the case for the $\mathrm{H}_{2}$ stiffnite deposit (after Lebreiro et al. 1997, who however interpret $\mathrm{H}_{2}$ as a turbidite deposit, as already mentioned). Those oxidation fronts appear to be related to high organic carbon contents [Lebreiro et al. 1998].

Let me add me a few words about the oxidation fronts outside a stiffnite: (i) electrical charges of triboplasma are present in a stiffnite; (ii) during stiffnite motion, and possibly to a greater extent after the emplacement of a stiffnite deposit, when the sediments are at rest, the triboplasma has the chance to chemically oxidize sea-floor sediments located below a stiffnite deposit, because triboplasma diffuses and the triboplasma particles have a net positive charge at the base of a stiffnite (i.e. at the base of sheet $\mathcal{L}^{+}$).

\subsection{The giant electric permittivity of a flowing stiffnite}

Within a stiffnite body in a submarine environment, charge separation of grains and triboplasma is possible if the electric field effects are negligible or small compared to the Lorentz forces. In this section, I discuss the negligible effects of electrical forces.

In a medium with relative electric permittivity $\varepsilon_{r}$, the Coulomb force $F_{e}$ (modulus) attracting two elementary charges $q\left(=1.6 \times 10^{-19} \mathrm{C}\right)$ and $-q$, at a distance $\Delta d$ apart from each other, is:

$$
F_{e}=\frac{q^{2}}{4 \pi \varepsilon_{0} \varepsilon_{r} \Delta d^{2}}
$$

where $\varepsilon_{0}$ is the electric permittivity of vacuum $\left(=8.85 \times 10^{-12}\right.$ $\mathrm{C}^{2} \mathrm{~N}^{-1} \mathrm{~m}^{-2}$ ). Let us say that those two charges of triboplsma were produced during a specific event of grain fragmentation. Initially those charges are very close each other, i.e., let us say, $\Delta d \approx 10^{-9} \mathrm{~m}$ (the dimension of a molecule). Let us also point out those two charges are located inside the stiffnite, which is moving at a velocity $u=10 \mathrm{~m} / \mathrm{s}$ to $100 \mathrm{~m} / \mathrm{s}$. Moreover, those charges are in a magnetic field $B_{\text {dip }}$ of $1 \mathrm{~T}$ to $10 \mathrm{~T}$. The magnetic force $\left(=q u B_{\text {dip }}\right)$ on those charges is given by Equation (1). Those two charges of triboplasma go away 
from each other, under the influence of Lorentz forces, if the attractive electrical force between them is negligible respect to magnetic Lorentz ones, i.e., if the medium between those two charges has a relative electric permittivity:

$$
\varepsilon_{r}>10^{-6} \div 10^{-8} \mathrm{SI}
$$

Some indirect information on the electric permittivity of natural mixtures of marine under-shear sediments containing shell fragments and other hard grains and polymers can be speculated upon from studies in materials science that deal with the insulator capacities of ceramics.

The ceramic framework is apparently different: (i) in laboratory experiments on materials, the specimens are at rest (no shear); (ii) a variable electrical field is applied; (iii) a theoretical assessment for the enhanced giant dielectric permeability in heterogeneous ceramic media is still lacking. Studies on piezoelectric and pyroelectric composites have recently stated that ferromagnetic ceramic powders dispersed in a polymer matrix have giant dielectric properties, with typical relative electric permittivities of $10^{7}$ to $10^{8}$, or even larger [Saha 2008, and references therein]. Studies on nanodielectrics by Turik et al. [2004] and Saha [2008] deal with composite materials in which nano-sized ceramic particles are embedded in insulating matrices. Most of the models developed to explain the dielectric and electroactive properties of composites are based on a cube of unit dimensions that represents the matrix, with fractional inclusions that represent the ceramic [Newnham et al. 1978]. For composites consisting of a ceramic and a polymer phase, there are ten possible combinations of phase connectivity [Wenger and Das-Gupta 1999]. These are indicated by $\mathrm{n}-\mathrm{m}$, the first digit $\mathrm{n}$ denoting the self-connectivity of the ceramic, and the second (m), that of the polymer [Newnham et al. 1978]. A ceramic powder randomly dispersed in a polymer matrix is referred to as having a connectivity of 0 3 , i.e. the ceramic phase has no intraconnectivity in the $x, y$, and $z$ directions of the composite, whereas the polymers of the matrix have full intraconnectivity in these directions. In industrial applications, composites of 1-3 connectivity tend to have high piezoelectric and electromechanical coupling coefficients, although they are difficult and expensive to fabricate; the 0-3 composites that consist of ceramic powder dispersed in a polymer matrix, on the other hand, are relatively simple to produce and lend themselves to the formation of thin films. The connectivity of mixed connectivity composites ranges from 1-3 to 0-3 [Dias and Das-Gupta 1996]. Mixedconnectivity composite synthetic films show enhanced piezoelectric properties with respect to $0-3$ connectivity composites while remaining easy to fabricate and maintaining mechanical flexibility.

As a whole, suitably connected synthetic mixtures of polymers and ferromagnetic ceramic powders have a relative electric permittivity similar to those required by inequality
(23) in stiffnites.

Also if the shear does not characterize experiments with synthetic mixtures in nanotechnologies [Turik et al. 2004, Saha 2008], and, on the other hand, no variable electrical field characterizes a stiffnite, synthetic mixtures and our marine sediments have some common characteristics: the presence of polymers and 'permanent' magnetic dipoles. Moreover 'variable also if differently variable' factors occur in both the cases.

Let me discuss polymers in marine sediments. Lipids are fatty acid esters, which are a class of water-insoluble organic molecules that store energy and constitute the basic building blocks of biological membranes. Lipids consist of a polar or hydrophilic (attracted to water) 'head' and a hydrophobic (repelled by water) 'tail'. The hydrophobic tail consists of one or more fatty acids. Fatty acids are unbranched chains of carbon atoms that are connected by single bonds alone (saturated fatty acids) or by both single and double bonds (unsaturated fatty acids). For example, alkenones are unsaturated fatty acids that are biosynthesized by Emiliana huxleyi [Volkman et al. 1998, and references therein]; moreover, as already mentioned, these lipids can survive for millions of years in marine sediments, and therefore they can have active roles in a stiffnite for a long time after the death of the algae. More in general, marine lipids are produced by haptophytes, eustigmatophytes, dinoflagellates, archae and diatoms; cholesterol can also be present due to zooplankton herbivory [Schefuss et al. 2001]. However, whatever its nature, a lipid is a polymer too. Indeed, according to its definition, a polymer is a large molecule that is composed of repeating structural units that are typically connected by covalent chemical bonds. In addition, marine lipids are not the only polymers in marine deposits.

\subsection{Faraday forces \\ explain the peak of magnetic susceptibility}

As listed in Section 4, stiffnite deposits show a peak in magnetic susceptibility. Here I explain how this occurs.

Let us consider a grain of volume $V_{o l}$, and density $\rho_{f c}^{\prime}$ with ferromagnetic inclusions and average (ferromagnetic) susceptibility $\chi^{f e}$, in the 'frozen' magnetic field $\vec{B}_{\text {dip }}$, created by the Ambigua magnetic dipoles. At first, let us suppose a ferromagnetic grain with no electrical charges. This ferromagnetic grain acquires a moment $\vec{m}$ in the direction of the magnetic field $\vec{B}_{\text {dip }} ; \vec{m}$ is directed along the $x$-axis and has a size of:

$$
m_{x}=\frac{V_{o l} \chi^{f e} B_{d i p_{x}}}{\mu_{o}}
$$

where suffix $x$ indicates the component of the correspondent vector along the $x$-axis.

Both the grain magnetic moment and the spatial variability of the magnetic field produce a Faraday force on that grain. The usual electric current loop model for a 
magnetic dipole leads to the force $\vec{F}_{F a r}=\nabla(\vec{m} \cdot \vec{B})$ on a magnetic dipole in a magnetic field $\vec{B}$ [Boyer 1988]. In the stiffnite geometry of Figure 6i, the Faraday force $\vec{F}_{F a r}$ acting on a grain is directed along the $z$-axis:

$$
\vec{F}_{F a r}=\nabla\left(\vec{m} \cdot \vec{B}_{\text {dip }}\right)=\left(0,0, F_{\text {Far }_{z}}\right)=\left(0,0, \frac{d\left(m_{x} B_{\text {dipx }}\right)}{d z}\right)
$$

where according to Equation (24):

$$
F_{F a r_{z}}=\frac{d\left(m_{x} B_{d i p_{x}}\right)}{d z}=\frac{2 V_{o l} \chi^{f e} B_{d i p_{x}}}{\mu_{0}} \frac{d B_{d i p_{x}}}{d z}
$$

It is interesting to note that the Faraday force always points downwards, because the gradient of the magnetic field is negative along the $z$-axis according to Equations (11) and (12). Moreover, the ratio $R_{a t}$ between Faraday and gravity forces acting on a grain is:

$$
R_{a t}=2 \frac{\chi^{f e} B_{d i p_{x}}}{\rho_{f e}^{\prime} g \mu_{0}}\left|\frac{d B_{d i p_{x}}}{d z}\right|
$$

If, for example, we consider $B_{\text {dipx }}$ ca. $1 \mathrm{~T}$ to $10 \mathrm{~T}$, $\left|d B_{d i p_{x}} / d z\right|$ ca. $10 \mathrm{~T} / \mathrm{m}$ to $100 \mathrm{~T} / \mathrm{m}$, density $\rho_{f e}^{\prime}$ ca. $10^{3}$ $\mathrm{kg} / \mathrm{m}^{3}, \chi^{\text {fe }}$ ca. $10^{-2} \mathrm{SI}$ (see Section 4.2.2), it follows that $R_{a t}$ is ca. 10 to $10^{3}$. It is interesting to note that $R_{a t}$ does not depend on the size of the grain. For grains with sufficiently high ferromagnetic inclusions, Faraday forces prevail over gravity.

As a whole, the Faraday forces introduce a sorting of the ferromagnetic electrically neutral grains. Let us now discuss the case of electrified grains. Although the Faraday forces tend to displace ferromagnetic grains downwards, towards the lower boundary of a stiffnite, the peak of the magnetic susceptibility might not occur at the base of the flow. To support this consideration, let us consider the following two points. Both of these points are related to the role of the Lorentz forces with respect to the Faraday forces.

First of all, iron minerals on the external surface of a grain can reduce the grain propensity to triboelectric exchanges. Fe and steel powders occupy neutral positions in the triboelectric scale, in contrast to other metals that are negative in this scale [Shaw 1917, Adams 1987]. That is, Festeel grains have no tendency to exchange charges at a triboelectric contact. Also, if in marine deposits, ferromagnetic grains are not homogeneously made by iron or steel, it is possible that the greater the ferromagnetic susceptibility of a grain (i.e. its iron/steel content), the lower the triboelectric charge acquired by that grain. In other words, on average, paramagnetic microshells fragments can be characterized by higher triboelectric charges than ferromagnetic grains of the same dimensions.

For the second point: for a negative ferromagnetic grain, the Lorentz and Faraday forces are both vertical, but they have opposite directions.

As a whole, the magnetic susceptibility can acquire a peaked distribution under the effects of Ambigua.
From Equation (24), the magnetic dipole $m$ is $10^{-15} \mathrm{~A} \mathrm{~m}^{2}$ to $10^{-13} \mathrm{~A} \mathrm{~m}^{2}$ for a grain volume of $10^{-18} \mathrm{~m}^{3}(1 \mu \mathrm{m}$ in diameter), a magnetic susceptibility of $10^{-3} \mathrm{SI}$ to $10^{-2} \mathrm{SI}$, and a magnetic field of $1 \mathrm{~T}$ to $10 \mathrm{~T}$.

Now, let me consider the correlation between the assumption of a 'frozen' vertical position of Ambigua and the peaked distribution/displacements of ferromagnetic grains inside a stiffnite. In the presence of the magnetic field created by Ambigua, inside a stiffnite the total magnetic field is:

$$
\vec{B}_{T}=\vec{B}_{d i p}+\chi_{m}^{f e} \vec{B}_{d i p}+\chi_{m}^{s h} \vec{B}_{d i p}
$$

where $\chi_{m}^{f e}$ is the average magnetic susceptibility of a stiffnite due to the ferromagnetic grains, and $\chi_{m}^{s h}$ is that due to the paramagnetic microshells/ or other constituents. Let us consider: (i) $B_{\text {dip }}=1 \mathrm{~T}$ to $10 \mathrm{~T}$; (ii) $\chi_{m^{f e}}^{\text {ce }}$ ca. $10^{-3} \mathrm{SI}$ (or lower) at the magnetic susceptibility peak inside a stiffnite; and (iii) the typical background values of magnetic susceptibility $\chi_{m}^{\text {sh }}$ are $10^{-4} \mathrm{SI}$ (or lower) (see Section 4). From Equation (28), it follows that (on the average) $\vec{B}_{T} \approx \vec{B}_{d i p}$. In other words, (generally) the ferromagnetic grains, and with more reason, the paramagnetic microshells do not significantly affect the magnetic field $\vec{B}_{d i p}$ that is created by Ambigua. Consequently, no internal inconsistencies contradict the hypothesis relating to the frozen vertical position of Ambigua. I will reconsider this point in the next section.

\subsection{About the nature of stiffnite magnetic dipoles}

Can we tell something about the magnetic dipoles Ambigua?

\subsubsection{Preliminary considerations}

Let me consider the following points.

Point 1. Whatever its relativistic or classical explanation, a current always implies two sets of charges with opposite polarities, with these sets moving with respect each others (Massimiliano Favalli, private communication). For example, in an atom, electrons 'rotate' around the nucleus of protons (and neutrons), or free electrons move through the standing lattice of a metallic conductor.

Point 2. Lorentz forces act on free electrons and force them to move around a closed circle in an external magnetic field. The point is that we are just looking for the trigger of that 'external' field.

Point 3. In the experiments of Saha [2008], the two main ingredients that characterize a mixture of giant permittivity are ferromagnetic powders and polymers. By analogy, in a stiffnite, we can argue that a high giant permittivity occurs because the 'fabric' includes marine polymers and magnetic dipoles. However, the ferromagnetic particles of a stiffnite cannot be the magnetic dipoles Ambigua. Ferromagnetic fragments move vertically under the influence of Ambigua, as supported by the occurrence of a peak of magnetic 
susceptibility in stiffnite deposits. In other words, ferromagnetic grains are not frozen inside a stiffnite.

Point 4. According to Equation (24), typical values of the magnetic moment of ferromagnetic particles are $10^{-15} \mathrm{~A} \mathrm{~m}^{2}$ to $10^{-13} \mathrm{~A} \mathrm{~m}^{2}$. The magnetic dipoles of Ambigua must be much greater than those of ferromagnetic particles, because Ambigua are frozen with respect to ferromagnetic grains and must dislocate these ferromagnetic grains. According to this constraint, a lower limit of the average magnetic dipole $\bar{\mu}$ of Ambigua in marine stiffnites would be $10^{-12} \mathrm{~A} \mathrm{~m}^{2}$ to $10^{-11} \mathrm{~A} \mathrm{~m}^{2}$.

According to Equation (13), if we assume that $B_{\text {dip }}$ is ca. $1 \mathrm{~T}$ to $10 \mathrm{~T}$, it follows that: [mean value of the product $\mu \mathrm{N}$ ] $=\mathrm{ca} .10^{6} \mathrm{Am}^{-1}$ to $10^{7} \mathrm{Am}^{-1}$. If we can neglect covariance, we can replace the [mean value of the product $\mu N$ ] with the product of the mean values $\bar{\mu}$ and $\bar{N}$, so that:

$$
\bar{\mu} \overline{\mathrm{N}} \text { ca. } 10^{6} \mathrm{Am}^{-1} \text { to } 10^{7} \mathrm{Am}^{-1}
$$

where $\bar{N}$ is the average density of Ambigua and $\bar{\mu}$ is the (average) magnetic dipole of an Ambiguum. From Equation (29) and from $\bar{\mu}$ ca. $10^{-12} \mathrm{~A} \mathrm{~m}^{2}$ to $10^{-11} \mathrm{~A} \mathrm{~m}^{2}$, it follows that $\bar{N}$ is $10^{17}$ to $10^{19}$ Ambigua per cubic meter.

In marine sediments, the upper concentration of the lipid molecules is $10^{26}$ molecules $/ \mathrm{m}^{3}$. This is an upper boundary that I derived as a concentration from the estimated total organic carbon contents of $10 \mathrm{wt} \%$ in marine cores [Schefuss et al. 2001] and from the assumptions that all of this organic carbon belongs to the lipid molecules, and that the average number of carbon atoms in a polymer is 35 . From this value of $10^{26}$ molecules $/ \mathrm{m}^{3}$, it follows that lipids can contribute to the formation of Ambigua because there are $\bar{N}=10^{17}$ to $10^{19}$ Ambigua per cubic meter, on average. Their abundance allows this. Other nonlipid polymer molecules could also be present.

Point 5 . The shear rate is also an ingredient of our stiffnite cocktail, because Ambigua (their magnetic moments) disappear (almost everywhere) in stiffnite deposits at rest. It is interesting to consider that electrons generate a counter-clockwise current if they run clockwise around a closed loop about a some sort of 'atomic' heart (looking at them from the positive $x$-axis). The horizontal velocity of a stiffnite increases with $z$ (Figures $5 c, 6 i)$. As a consequence, the horizontal velocity of a stiffnite has the right trend to 'efficiently push' an electron according to a clockwise motion along a closed loop if the axis of this loop is parallel to the $x$-axis (the area of the loop lies in a $z-y$ plane, Figure 6i), and if this loop is elongated along the $y$-axis. Moreover, if that loop is elongated along the $y$-axis it offers minimum resistance to a down-slope motion. Last, but not least, the magnetic moment that is created by a current of negative electrons along the loop is directed along the positive $x$-axis, as we wish for the magnetic moment $\mu$ of an Ambiguum.

Point 6. Shear polymer media have intriguing behaviors. Experiments by Feng and Joseph [1996, and references therein] showed that in a shearing torsional flow of polymer melts with suspensions of glass beads or short fibers, spheres form chains and aggregates. The chains are along the flow direction and can connect to form circular rings. Rings migrate outwards at a velocity much higher than that of a single sphere. Feng and Joseph [1996] also used rods. Those elongated particles aggregate in much the same way, alhough to a lesser extent than spheres. In the experiments by Feng and Joseph (1996), the sphere diameters were $250 \mu \mathrm{m}$ to 850 $\mathrm{mm}$; the rod diameters $170 \mu \mathrm{m}$ to $330 \mu \mathrm{m}$, and the rod lengths $0.5 \mathrm{~mm}$ to $4.0 \mathrm{~mm}$. However, the dimensions of the rods of Feng and Joseph [1996] are significantly greater than those of the rod-shaped polymer molecules (by a factor of $10^{5}$ ): for example, an alkenon is $2 \mathrm{~nm}$ long, with a diameter of $0.5 \mathrm{~nm}$.

\subsubsection{The nature of Ambigua}

The above-listed points 1-6 are satisfied if I assume that an Ambiguum is a string of aligned polymers that are stretched along the $y$-axis (i.e. along a streamline of the stiffnite flow). The polymers are linked to each other at their extremities, to form a chain. The polymers of each string share some of their 'external' electrons, which are free to run along the external perimeter of the string as along a closed track. Looking to the string from the positive $x$-axis, the shared electrons of the polymers run from left to right in the upper side of a string (where the velocities of the medium are higher) and come back from right to left at the lower side of this string, where the velocities of the medium are lower. In other words, electrons of each string run clockwise along a closed track, looking at the loop from the positive $x$-axis.

Let us suppose that an Ambiguum formed by a chain of aligned polymers has length $L_{G}$, along the $y$-axis. It follows that:

$$
L_{G}=n_{p o} l_{p o}
$$

where $l_{p o}$ is the length of a polymer of ca. $2 \mathrm{~nm}$, and $n_{p o}$ is the number of polymers in an Ambiguum. Equation (30) holds for (depth) averaged values $\bar{n}_{p o}$ and $\bar{L}_{G}$ too.

From the definition of magnetic moment, the Ambigua average magnetic dipoles $\bar{\mu}$ are given by:

$$
\begin{gathered}
\bar{\mu}=(\text { loop area }) \cdot(\text { current along the loop })= \\
=\bar{L}_{G} w_{p o} \cdot(\text { current along the loop })
\end{gathered}
$$

where $w_{p o}$ is the width of a polymer molecule (ca. $0.5 \mathrm{~nm}$ ). From Equations (30) and (31), it follows that the average number $\bar{n}_{p o}$ of polymers in an Ambiguum is:

$$
\bar{n}_{p o}=\frac{2 \bar{\mu}}{w_{p o} 10^{2} q v_{e l}} \sim 10^{8} \text { to } 10^{9}
$$


where $q$ the absolute value of the charge of an electron $=$ $1.6 \times 10^{-19} \mathrm{C}$, and $\bar{\mu}=10^{-12} \mathrm{~A} \mathrm{~m}^{2}$ to $10^{-11} \mathrm{~A} \mathrm{~m}^{2}$, as considered above in Point 4. In Equation (32), I also fixed that: (i) each polymer of the string shares, let us say, ca. $10^{2}$ electrons to the string, so that the average total number of shared electrons in a string is: $\bar{n}_{p o} 10^{2}$; (ii) the shared electrons of the polymers of the string move along the closed string at their atomic velocity $v_{e l}=10^{6} \mathrm{~m} / \mathrm{s}$.

From Equations (30) and (32), the average length of an Ambiguum is $\bar{L}_{G}=0.1 \mathrm{~m}$ to $1 \mathrm{~m}$, and the total number of shared electrons in a string is $=10^{10}$ to $10^{11}$.

Moreover, if all of the polymers are used to build Ambigua, the number $N_{T p o}$ of polymer molecules per unit volume in a stiffnite medium (which is a given value in virgin marine sediments) must be:

$$
N_{\text {Tpo }}=\bar{n}_{p o} \bar{N} \sim 10^{25} \text { to } 10^{28} \mathrm{~m}^{-3}
$$

which is roughly compatible with/bounded from above by the number $10^{26} \mathrm{~m}^{-3}$ of marine lipid molecules from field data, as discussed in Point 4.

This very long length of an Ambiguum in the direction of stiffnite motion explains the frozen hypothesis of Ambigua inside a stiffnite. Ambigua are so long with respect to the other particles of a stiffnite that they follow stiffnite streamlines and they are forced to maintain that path by many packed, contiguous, much-smaller stiffnite grains.

In a stiffnite, Ambigua are frozen. However, in an immature stiffnite, Ambigua, smaller then local grain sizes, can migrate and merge in longer strings.

An Ambiguum, sandwiched between electrical charges, isolate those charges.
Let us now discuss the linear dependence of magnetization $M$ (and consequently of the magnetic field $B_{\text {dip }}$ ) on the shear rate, as introduced in Section 5.3.

The magnetic dipole moment $\mu$ of an Ambiguum is proportional to $n_{p o}$, according to Equation (32). Magnetization $M$ is given by $N \mu$, according to Equation (10). It follows that the magnetization $M$ is proportional to the density $N$ of Ambigua times the number of aligned molecules $n_{p o}$ that form an Ambiguum, i.e. magnetization $M$ is proportional to $N_{T p o}$. As I have assumed that the magnetization is proportional to the shear rate, it follows that $N_{T p o}$ is proportional to the shear rate.

This means that polymers spatially redistribute themselves during the immature stiffnite phase.

It is possible that, in a stiffnite deposit at rest, almost all Ambigua disappear for relaxation, with the exception of those Ambigua very close to ferromagnetic particles, in turn those ferromagnetic particles permanently magnetized by Ambigua during stiffnite motion.

I call the string of polymers with a global magnified magnetic dipole moment an Ambiguum, as was suggested to me.

\section{Conclusions}

Stiffnites are magnetically hardened sheets of sediments that move down-slope on the sea floor, under shear conditions.

Some of the constituents of stiffnites are: (i) polymers; (ii) 'hard' triboelectrically electrified grains (for example, calcite microshells of marine ooze); (iii) electrons and ions (= triboplasma) are produced at triboelectric contacts.

Magnetic dipoles Ambigua develop inside a stiffnite because of filiform polymers under shear conditions. Ambigua create a magnetic field $\vec{B}_{d i p}$. This magnetic field $\vec{B}_{d i p}$

\section{NOMENCLATURE}

\begin{tabular}{ccll}
\hline Name & Unit & Meaning \\
\hline$\vec{B}, B$ & $\mathrm{~T}$ & Magnetic field, modulus of magnetic field \\
$B_{1}, B_{2}, B_{3}$ & $\mathrm{~T}$ & Magnetic fields created by current densities $J_{1}, J_{2}, J_{3}$ respectively in sheets $\mathcal{L}_{1}, \mathcal{L}_{2}, \mathcal{L}_{3}$ \\
$\vec{B}_{\text {dip }}, B_{\text {dip }}$ & $\mathrm{T}$ & Magnetic field created by magnetic dipoles Ambigua, modulus of $\vec{B}_{\text {dip }}$ \\
$B_{\text {dip } x}$ & $\mathrm{~T}$ & Component along the $x$-axis of the magnetic field $\vec{B}_{\text {dip. }}$. In a stiffnite $\vec{B}_{\text {dip }}=\left(B_{d i p}, 0,0\right)$. \\
$B_{\text {dipmax }}$ & $\mathrm{T}$ & Maximum value of the modulus of $\vec{B}_{\text {dip }}$ \\
$B_{\max }$ & $\mathrm{T}$ & Maximum value of magnetic field $\vec{B}_{\text {tot }}$ \\
$\vec{B}_{T}$ & $\mathrm{~T}$ & Total magnetic field in a stiffnite (see Equation 28$)$ \\
$\vec{B}_{\text {tot }}$ & $\mathrm{T}$ & Magnetic field (see Section 5.3 .2$)$ \\
$d u / d z$ & $\mathrm{~s}^{-1}$ & Modulus of shear rate. $u$ is the stiffnite velocity along $y$ \\
$d z$ & $\mathrm{~m}$ & Infinitesimal thickness of a stratum \\
$E_{S S}$ & & Static Stability number, used to estimate the vertical stability of a motionless fluid \\
$F_{e}$ & $\mathrm{~N}$ & Electrical force (modulus) \\
$\vec{F}_{F a r}$ & $\mathrm{~N}$ & Faraday force \\
\hline
\end{tabular}




\section{NOMENCLATURE}

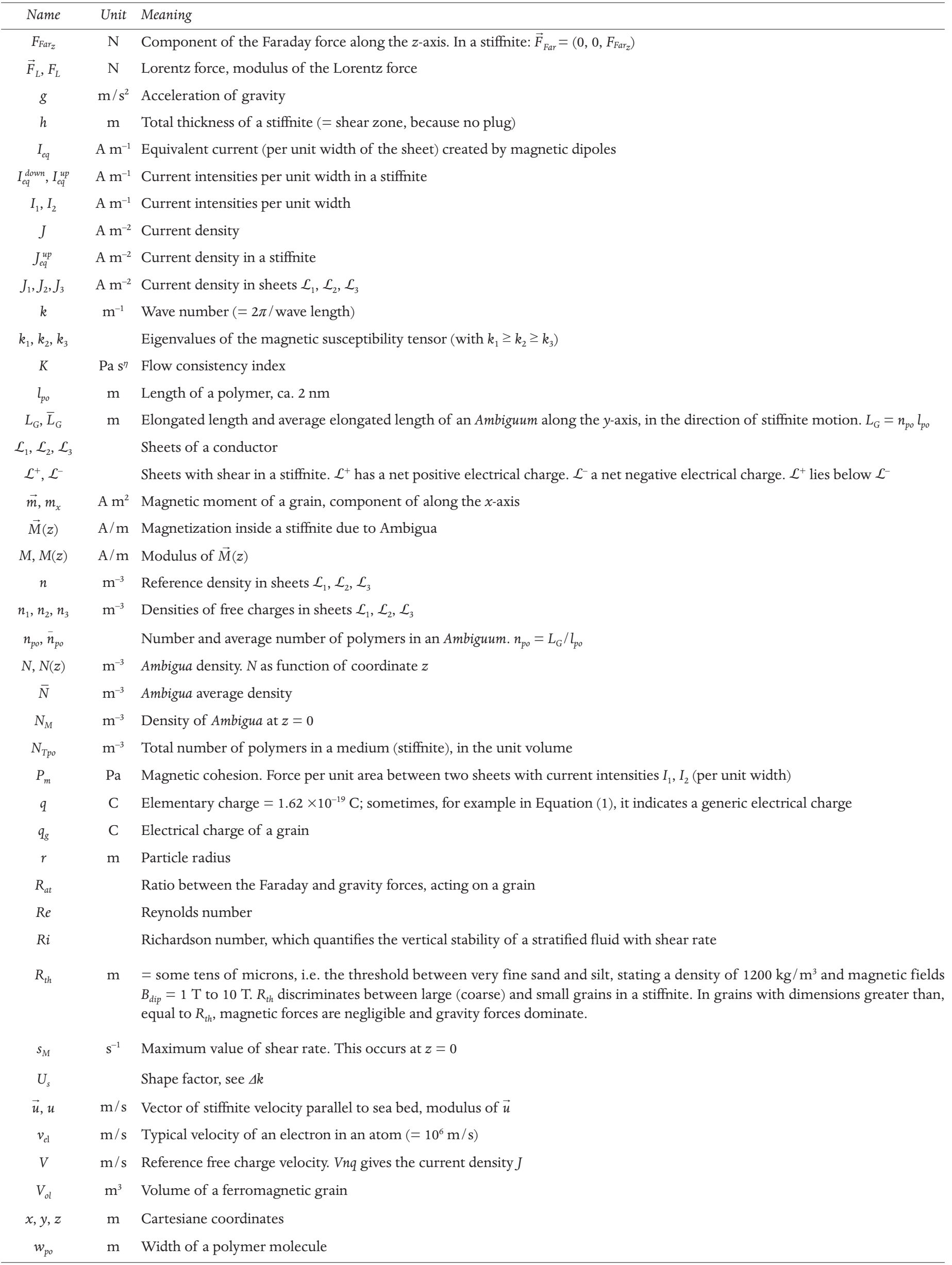




\section{GREEK SYMBOLS}

\begin{tabular}{|c|c|c|}
\hline Name & Unit & Meaning \\
\hline$\alpha$ & $\mathrm{kg} / \mathrm{C}$ & Constant of proportionality in Equation (19) \\
\hline$\partial u / \partial z$ & $\mathrm{~s}^{-1}$ & Shear rate. Here the same as $d u / d z$ \\
\hline$\delta$ & $\mathrm{m}$ & Reference thickness in Table 2, or thickness of a layer ten lines or so after Equation (21) \\
\hline$\Delta d$ & $\mathrm{~m}$ & Distance between two charges \\
\hline$\Delta \mathrm{H}$ & $\mathrm{m}$ & Height of climbed submarine relief \\
\hline$\Delta k=k_{1}-k_{3}$ & & Susceptibility difference, $\Delta k=k_{1}-k_{3}$. This gives a quantitative idea of anisotropy. \\
\hline$\Delta \rho_{\text {eq }}$ & $\mathrm{kg} / \mathrm{m}^{3}$ & Equivalent variation in the density of an electrified grain, due to the Lorentz force (see Equation 20) \\
\hline$\Delta U$ & $\mathrm{~m} / \mathrm{s}$ & Difference of velocities of two superimposed layers \\
\hline$\Delta z$ & $\mathrm{~m}$ & Thickness of a sheet \\
\hline$\Delta z_{1}, \Delta z_{2}, \Delta z_{3}$ & $\mathrm{~m}$ & Thickness of sheets $\mathcal{L}_{1}, \mathcal{L}_{2}, \mathcal{L}_{3}$ \\
\hline$\varepsilon_{0}$ & $\mathrm{C}^{2} \mathrm{~N}^{-1} \mathrm{~m}^{-2}$ & Dielectric permittivity of vacuum $=8.85 \times 10^{-12} \mathrm{C}^{2} \mathrm{~N}^{-1} \mathrm{~m}^{-2}$ \\
\hline$\varepsilon_{r}$ & & Relative electric permittivity \\
\hline$\eta$ & & Flow behavior index \\
\hline$\theta$ & & Bed slope angle \\
\hline$\vec{\mu}, \mu$ & $\mathrm{A} \mathrm{m} \mathrm{m}^{2}$ & Vector of magnetic dipole moment of an Ambiguum; modulus of $\vec{\mu}$ \\
\hline $\bar{\mu}$ & $\mathrm{A} \mathrm{m} \mathrm{m}^{2}$ & Average magnetic dipole moment of an Ambiguum (depth averaged value) \\
\hline$\mu_{e f f}$ & Pa s & $=K(\partial u / \partial z)^{\eta-1}$, apparent or effective viscosity as a function of the shear rate \\
\hline$\mu_{\mathrm{M}}$ & $\mathrm{A} \mathrm{m}^{2}$ & Dipole moment of an Ambiguum at $z=0$ \\
\hline$\mu_{o}$ & $\mathrm{~T} \mathrm{~m} / \mathrm{A}$ & Permeability of free space $=4 \pi \times 10^{-7} \mathrm{Tm} / \mathrm{A}$ \\
\hline$\mu_{r}$ & & Magnetic permittivity or permeability. $\mu_{r}=1+\chi_{m}$ \\
\hline$\mu_{S T}$ & Pa s & Stiffnite viscosity \\
\hline$\rho_{g}$ & $\mathrm{~kg} / \mathrm{m}^{3}$ & Grain density \\
\hline$\rho_{w}$ & $\mathrm{~kg} / \mathrm{m}^{3}$ & Water density \\
\hline$\rho$ & $\mathrm{kg} / \mathrm{m}^{3}$ & Stiffnite equivalent density \\
\hline$\rho^{\prime}$ & $\mathrm{kg} / \mathrm{m}^{3}$ & Submerged density of sediments (actual total density minus water density $\rho_{w}$ ) \\
\hline$\rho_{f e}^{\prime}$ & $\mathrm{kg} / \mathrm{m}^{3}$ & Density of a ferromagnetic grain \\
\hline$\sigma$ & $\mathrm{C} / \mathrm{m}^{2}$ & Charge density per unit surface (on the plate of the capacitor) \\
\hline$\tau$ & $\mathrm{Pa}$ & Shear stress \\
\hline$\tau_{o}$ & $\mathrm{~Pa}$ & Yield strength \\
\hline$\chi_{m}$ & & Magnetic susceptibility \\
\hline$\chi_{m}^{s h}$ & & $\begin{array}{l}\text { Average magnetic susceptibility of a stiffnite due to the contribution of microshells or microshell fragments and other not } \\
\text { ferromagnetic constituents }\end{array}$ \\
\hline$\chi_{m}^{f e}$ & & $\begin{array}{l}\text { Average magnetic susceptibility of stiffnites due to the contribution of volcanic particles and/or other particles with } \\
\text { ferromagnetic components }\end{array}$ \\
\hline$\chi^{f e}$ & & Magnetic susceptibility of a «ferromagnetic» grain \\
\hline
\end{tabular}

lies in the local shear plane and it is perpendicular to the direction of stiffnite motion.

At a worldwide insight, stiffnites occur in different frameworks that are characterized by different topographies, strengths of the trigger, thicknesses of the deposits, sea-floor depths and slopes, among other factors.

As a consequence, the main macroscopic instabilities (Kelvin Helmoltz; e.g. pinch, bending) appear negligible over large areas, above all if we consider: (i) the very thin vertical dimensions of stiffnites; and ii) that stiffnites move as a sandwiched sheet between two media at rest (sea water above, and usually water-saturated, weakly cohesive seafloor sediments below). This implies that 'cohesive' magnetic pressures created by Ambigua have a global stabilizing effect, in spite of the high inferred stiffnite velocities.

The reconstruction of stiffnite dynamics presented in this report is based on: comparisons among worldwide field data (from the literature), laboratory experiments on materials (from the literature) and order-of-magnitudequantified estimations. 


\section{References}

Abineri, K.W. (1999). (c) Microfossil and Nannofossil Image Gallery, http://www.microscopy-uk.org.uk/mag/indexmag.html?http: / / www.microscopy-uk.org.uk/ mag/artoct99/kamast5.html.

Abrantes, F., S. Lebreiro, T. Rodrigues, I. Gil, H. Bartels-Jónsdóttir, P. Oliveira, C. Kissel and J.O. Grimalt (2005). Shallow-marine sediment cores record climate variability and earthquake activity off Lisbon (Portugal) for the last 2000 years, Quat. Science Rev., 24, 2477-2494.

Abrantes, F., U. Alt-Epping, S. Lebreiro, A. Voelker, and R. Schneider (2008). Sedimentological record of tsunamis on shallow-shelf areas: the case of the 1969 AD and 1755 AD tsunamis on the Portuguese shelf off Lisbon, Mar. Geol., 249, 283-293.

Ashcroft, N.W., and N.D. Mermin (1976). Solid State Physics, Holt Rinehart \& Winston, Forth Worth, Texas.

Boyer, T.H. (1988). The force on a magnetic dipole, Am. J. Phys., 56, 8, 688-692.

Defina, A., S. Lanzoni, F.M. Susin (1999). Stabiliy of a statified viscous shear flow in a tilted tube, Phys. Fluids, 11, 344-355.

Dias, C.J. and D.K. Das-Gupta (1996). Inorganic ceramic/ polymer ferroelectric composite electrets, IEEE Trans. Dielectrics and Electrical Insulation, 3, 706-734.

Farrel, W.M., P.H. Smith, G.T. Delory, G.B. Hillard, J.R. Marshall, D. Catling, M. Hecht, D.M. Tratt, N. Renno, M.D. Desch, S.A. Cummer, J.G. Houser and B. Johnson (2004). Electric and magnetic signatures of dust devils from the 2000-2001 MATADOR desert tests, J. Geoph. Res., 109, E03004; doi: 10.1029/2003JE002088.

Feng, J. and D.D. Joseph (1996). The motion of solid particles suspended in viscoelastic fluids under torsional shear, J. Fluid Mech., 324, 199-222.

Funada, T. and D.D. Joseph (2001). Viscous potential flow analysis of Kelvin-Helmholtz instability in a channel, J. Fluid Mech., 445, 263-283.

Garcia, M.O. and D.M. Hull (1994). Turbidites from giant Hawaiian landslides: Results from Ocean Drilling Program Site 842, Geology, 22, 159-162.

Garcia, M.O. (1996). Turbidites from failure on Hawaii volcanoes, in Volcano instability on the Earth and other planets, Geological Society of London Special Publication, vol. 110, edited by W.J. Mcguire, A.P. Jones and J. Neuberg, 281-294.

Haus, H.A. and J.R. Melcher (1990). Solutions manual for Electromagnetic Fields and Energy, MIT OpenCourseWare, http: / ocw.mit.edu, Prentice Hall, Englewood Cliffs, New Jersey 07632.

Hummel, R.H. (2000). Electronic properties of materials, 3rd ed., Springer-Verlag New York Berlin Heidelberg, $438 \mathrm{pp}$.

Inculet, I.I., G.S. P. Castle and G. Aartsen (2006). Generation of bipolar electric fields during industrial handling of powders, Chemical Engineering Science, 61, 2249-2253.

Jiménez-Munt, I., M. Fernàndez, M. Torne and P. Bird (2001). The transition from linear plate boundary in the Azores-Gibraltar region: results from a thin-sheet model, Earth Planet. Science Lett., 192, 175-189.

Lacks, D.J. and A. Levandovsky (2007). Effects of particle size distribution on the polarity of triboelectric charging in granular insulator systems, J. Electrostatics, 65 (2), 107-112.

Lanza, R. and A. Meloni (2006). The Earth's Magnetism, an Introduction for Geologists, Springer-Verlag Berlin Heidenberg, Germany, 278 pp.

Lebreiro, S.M., N. Mccave and P.P.E. Weaver (1997). Late Quaternary turbidite emplacement on the Horseshoe Abyssal Plain (Iberian Margin), J. Sed. Res., 67, 5, 856-870.

Lebreiro, S.M., P.P.E. Weaver and R.W. Howe (1998). Sedimentation of the Madeira Abyssal plain: Eocene-Pleistocene history of turbidite infill, in Proceedings of the Ocean drilling Program, Scientific Results, P.P.E, Weaver, H.U., Schmincke, J.W., Firth and W., Duffield, (Eds.), vol. 157, 523-531.

Masson, D.G. (1996). Catastrophic collapse of the volcanic island of Hierro $15 \mathrm{ka}$ ago and the history of landslides in the Canary Islands, Geology, 24, 231-234.

Miura T., T. Koyaguchi and Y. Tanaka (2002). Measurements of electric charge distribution in volcanic plumes at Sakurajima volcano, Japan, Bull. Volcanol., 64, 75-93; doi: 10.1007/s00445-001-0182-1.

Moore, J.G., D.A. Clague, R.T. Holcomb, P.W. Lipman, W.R. Normark and M.E. Torresan (1989). Prodigious submarine landslides on the Hawaiian ridge, J. Geoph. Res., 94, 17465-17484.

Morel, J.L and M. Meghraoui (1996). Gorige-Alboran-Tell tectonic zone: A transpression system along the AfricaEurasia plate boundary, Geology, 24, 755-758.

Nakayama, K. and J.M. Martin (2006). Triboelectrical reactions at and in the vivinity of a sliding contact, Wear, 261, 235-240.

Nakayama, K. (2007). The plasma generated and photon emitted in an-oil-lubrificated sliding contact, Journal of Physics D: Applied Physics, 40, 1103-1107.

Newnham, R.E., D.P. Skinner and L.E. Cross (1978). Connectivity and piezoelectric-pyroelectric composites, Material Res. Bull., 13, 525-536.

Pareschi, M.T., M. Favalli and E. Boschi (2006). Impact of the Minoan tsunami of Santorini: simulated scenarios in the Eastern Mediterranean, Geoph. Res. Lett., 33, L18607; doi: 10.1029/2006GL027205.

Pareschi, M.T. (2011). Stiffnites. Part I, Annals of Geophysics, this issue.

Pearse, M.J. and M.J. Pope (1977). The triboelectric separation of quartz-calcite and quartz-apatite powders 
after chemical conditioning, Powder Technology, 17, 83-89.

Rochette, P. (1988). Inverse magnetic fabric in carbonatebearing rocks, Earth Plan. Sc. Lett., 90, 229-237.

Saha, S.K. (2008). Nanodielectrics with giant permittivity, Bull. Mater. Science, 31, 3, 473-477.

Savage, S.B. and C.K.K. Lun (1988). Particle size segregation in inclined flow of dry cohesionless granular solids, J. Fluid Mech., 189, 311-335.

Schefuss, E., G.J.M. Versteegh, J.H.F. Jansen and J.S. Sinninghe Damsté (2001). Marine and terrigenous lipids in Southeast Atlantic Sediments (Leg 175) as paleoenvironmental indicators: initial results, Proceedings of the Ocean Drilling Program, Scientific Results, edited by G. Wefer, W.H. Berger and C. Richter, 175, 1-34 (online).

Schmidt, V., D. Gunther and A.M. Hirt (2006). Magnetic anisotropy of calcite at room-temperature, Tectonophysics, 418, 63-73.

Shaw, P.E. (1917). Experiments on Tribo-electricity. I.-The Tribo-electric series, Phil. Trans. A, 217, 16-33.

Stewart, R.H. (2005). Introduction to physical oceanography; http: / / oceanworld.tamu.edu/ resources/ocng_ textbook/chapter08/chapter08_04.htm.

Turik, A.V., G.S. Radchenko, A.I. Chernobabov and S.A. Turik (2004). Dielectric constant of polymer matrices containing isolated inclusions: giant dielectric enhancement instead of collective resonance, JETP Letters, 79 (9), 407-409.

Volkman, J.K., S.M. Barrett, S.I. Blackburn, M.P. Mansour, E.L. Sikes and F. Gelin (1998). Microalgal biomarkers: a review of recent research developments, Org. Geochem., 29 (5), 1163-1179.

Watt, A.B. and D.G.Masson (1995). A giant landslide on the northern flank of Tenerife, Canary Islands, J. Geoph. Res., 100, B12, 24,487-24498.

Weaver, P.P.E., I, Jarvis, S.M., Lebreiro, B., Alibés, J., Baraza, R., Howe and R.G. Rothwell (1998). Neogene turbidite sequence on the Madeira Abyssal Plain: Basin filling and diagenesis in the deep Ocean, in: Proceedings of the Ocean drilling Program, Scientific Results, edited by P.P.E Weaver, H.U. Schmincke, J.W. Firth and W, Duffield, vol. 157, 619-634.

Wenger, M.P. and D. K. Das-Gupta (1999). Mixed connectivity composite material characterization for electroactive sensors, Polymer Engineering \& Science, 39, 7, 1176-1188.

Wynn, R.B., P.P.E. Weaver, D.G. Masson and D.A.V. Stow (2002). Turbidite depositional architecture across three interconnected deep-water basins on the north-west African margin, Sedimentology, 49, 669-695.

Wynn, R.B. and D.G. Masson (2003). Canary islands landslides and tsunami generation: can we use turbidite deposits to interpret landslide processes, in Submarine
Mass Movements amd their consequences, edited by J. Locat and J. Mienert, Kluwer Academic Publishers, Dordrecht, Boston, London, 325-332.
${ }^{\star}$ Corresponding author: Maria Teresa Pareschi, Istituto Nazionale di Geofisica e Vulcanologia, Sezione di Pisa, Italy; e-mail: pareschi@pi.ingv.it.

C 2011 by the Istituto Nazionale di Geofisica e Vulcanologia. All rights reserved. 